ESAIM: COCV 21 (2015) 1178-1204

DOI: $10.1051 / \mathrm{cocv} / 2014063$
ESAIM: Control, Optimisation and Calculus of Variations

www.esaim-cocv.org

\title{
CONTROLLABILITY OF LINEAR AND SEMILINEAR NON-DIAGONALIZABLE PARABOLIC SYSTEMS *
}

\author{
Enrique Fernández-CarA ${ }^{1}$, Manuel GonzÁlez-Burgos ${ }^{1}$ And Luz de TeresA ${ }^{2}$
}

\begin{abstract}
This paper is concerned with the controllability of some (linear and semilinear) nondiagonalizable parabolic systems of PDEs. We will show that the well known null controllability properties of the classical heat equation are also satisfied by these systems at least when there are as many scalar controls as equations and some (maybe technical) conditions are satisfied. We will also show that, in some particular situations, the number of controls can be reduced. The minimal amount is then determined by a Kalman rank condition.
\end{abstract}

Mathematics Subject Classification. 93B05, 35K20.

Received March 15, 2012. Revised April 29, 2014.

Published online July 6, 2015.

\section{INTRODUCTION}

This paper deals with the controllability properties of some non-diagonalizable parabolic systems.

Let $\Omega \subset \mathbb{R}^{N}$ be a non-empty regular and bounded domain, let us fix $T>0$ and let us set $Q:=\Omega \times(0, T)$ and $\Sigma:=\partial \Omega \times(0, T)$. We will first consider the linear system

$$
\begin{cases}y_{t}-A \Delta y=M(x, t) y+B v 1_{\omega} & \text { in } Q, \\ y=0 & \text { on } \Sigma, \\ y(x, 0)=y_{0}(x) & \text { in } \Omega,\end{cases}
$$

where $\omega \subset \Omega$ is a (small) open subdomain,

$$
A \in \mathcal{L}\left(\mathbb{R}^{n}\right), M \in L^{\infty}\left(Q ; \mathcal{L}\left(\mathbb{R}^{n}\right)\right), B \in \mathcal{L}\left(\mathbb{R}^{n^{\prime}} ; \mathbb{R}^{n}\right) \text { and } y_{0} \in L^{2}\left(\Omega ; \mathbb{R}^{n}\right) .
$$

Here, $v=\left(v_{1}, \ldots, v_{n^{\prime}}\right)^{*}$ is the control, to be determined for example in $L^{2}\left(\omega \times(0, T) ; \mathbb{R}^{n^{\prime}}\right)$, while $y=$ $\left(y_{1}, \ldots, y_{n}\right)^{*}$ is the state. Of course, the most interesting situation appears when we are able to control the system for large $n$ and small $n^{\prime}$, since this means that we govern the behavior of many equations with few

Keywords and phrases. Null controllability, parabolic, non-diagonalizable.

* Supported by Grant MTM2010-15592 of D.G.E.S. (Spain) and project IN101013 of D.G.A.P.A. (Mexico).

1 Dpto, E.D.A.N., Universidad de Sevilla, Aptdo. 1160, 41080 Sevilla, Spain. cara@us.es \& manoloburgos@us.es

2 Instituto de Matemáticas, Universidad Nacional Autónoma de México, Circuito Exterior, C.U. 04510 D.F. México, Mexico.

ldeteresa@im.unam.mx 
controls. However, we will see that the coupling through the non-diagonalizable matrix $A$ in the higher order terms of the operator introduces serious difficulties to control with $n^{\prime}<n$.

The following assumption will be assumed throughout this paper:

$$
\exists a_{0}>0 \text { such that } A \xi \cdot \xi \geq a_{0}|\xi|^{2} \quad \forall \xi \in \mathbb{R}^{n} .
$$

Notice that, if $(1.2)$ is satisfied, for every $v \in L^{2}\left(\omega \times(0, T) ; \mathbb{R}^{n^{\prime}}\right)$ and every $y_{0} \in L^{2}\left(\Omega ; \mathbb{R}^{n}\right),(1.1)$ possesses a unique weak solution $y$, with

$$
y \in L^{2}\left(0, T ; H_{0}^{1}\left(\Omega ; \mathbb{R}^{n}\right)\right) \cap C^{0}\left([0, T] ; L^{2}\left(\Omega ; \mathbb{R}^{n}\right)\right) ;
$$

see Section 2.

For maybe technical reasons, we will also assume that

The dimensions of the Jordan blocks of the canonical form of $A$ are $\leq 4$.

It will be said that (1.1) is null-controllable at time $T$ if, for any $y_{0} \in L^{2}\left(\Omega ; \mathbb{R}^{n}\right)$, there exists $v \in L^{2}(\omega \times$ $\left.(0, T) ; \mathbb{R}^{n^{\prime}}\right)$ such that the associated solution satisfies

$$
y(x, T)=0 \text { in } \Omega .
$$

Since (1.1) is linear, this is equivalent to the exact controllability to the trajectories at time $T$, that is to say, to the following property: for any trajectory $\widehat{y}$ (i.e. any weak solution to (1.1) corresponding to an initial state $\widehat{y}_{0} \in L^{2}\left(\Omega ; \mathbb{R}^{n}\right)$ and the control $\left.v \equiv 0\right)$ and any $y_{0} \in L^{2}\left(\Omega ; \mathbb{R}^{n}\right)$, there exists $v \in L^{2}\left(\omega \times(0, T) ; \mathbb{R}^{n^{\prime}}\right)$ such that the associated solution satisfies

$$
y(x, T)=\widehat{y}(x, T) \text { in } \Omega .
$$

Consequently, the null controllability of (1.1) also implies approximate controllability, i.e. the fact that, for any $y_{0}, y_{d} \in L^{2}\left(\Omega ; \mathbb{R}^{n}\right)$ and any $\varepsilon>0$, there exists $v \in L^{2}\left(\omega \times(0, T) ; \mathbb{R}^{n^{\prime}}\right)$ such that the associated solution satisfies

$$
\left\|y(\cdot, T)-y_{d}\right\|_{L^{2}} \leq \varepsilon
$$

The controllability properties of similar scalar problems are nowadays well known; see for instance $[18,19,22,26,31,32]$. To be precise, let us consider the following control system

$$
\begin{cases}z_{t}-\Delta z=u 1_{\omega} & \text { in } Q \\ z=0 & \text { on } \Sigma, \\ z(x, 0)=z_{0}(x) & \text { in } \Omega .\end{cases}
$$

Then, for every $\Omega, \omega$ and $T,(1.6)$ is null-controllable at time $T$; see [26,31].

To our knowledge, almost all the papers in the literature devoted to the controllability of parabolic non-scalar systems of PDEs deal with distributed controls; see for instance [3-5,14,27-29,35]. In these papers, most results have been established for $2 \times 2$ systems, with the control exerted only on one equation. The best achievements in this context seem to be those in [4,5,28]. In [28], the authors study a cascade parabolic system of $n$ equations $(n \geq 2)$ controlled with one single distributed control. In $[4,5]$, the authors provide necessary and sufficient conditions for the controllability of $n \times n$ parabolic linear systems with constant or time-dependent coefficients. The analysis of similar boundary controllability problems has been the objective of $[7,13,20]$. A review of all these results can be found in [8].

It is an interesting fact that, in the framework of the controllability of coupled parabolic systems, new (and possibly counter-intuitive) phenomena arise: minimal time of controllability and dependence of the controllability result on the position of the control domain $\omega$; see [9-11,15]. 
Let us recall one of the main results proved in [4]. Consider the problem

$$
\begin{cases}y_{t}-\Delta y=M y+B v 1_{\omega} & \text { in } Q \\ y=0 & \text { on } \Sigma \\ y(\cdot, 0)=y_{0} & \text { in } \Omega\end{cases}
$$

where $M \in \mathcal{L}\left(\mathbb{R}^{n} ; \mathbb{R}^{n}\right), B \in \mathcal{L}\left(\mathbb{R}^{n^{\prime}} ; \mathbb{R}^{n}\right)$ (with $n, n^{\prime} \geq 1$ ) and $y_{0} \in L^{2}\left(\Omega ; \mathbb{R}^{n}\right)$. Let $[M \mid B]$ be the following matrix in $\mathcal{L}\left(\mathbb{R}^{n \times n^{\prime}} ; \mathbb{R}^{n}\right)$ :

$$
[M \mid B]=\left[B|M B| M^{2} B|\ldots| M^{n-1} B\right] .
$$

Then the following holds:

The linear system (1.7) is null controllable if and only the so called Kalman's rank condition

$$
\operatorname{rank}[M \mid B]=n
$$

is satisfied. In that case, null controllability holds at time $T>0$.

In this paper, our first main result is the following.

Theorem 1.1. Let $A, M=M(x, t)$ and $B$ be as above. Assume that (1.2) and (1.3) are satisfied and, also,

$$
n^{\prime} \geq n, \quad \operatorname{rank} B=n .
$$

Then (1.1) is null controllable.

In practice, (1.9) means that there are many scalar controls in the system (at least as many as scalar states) and, moreover, their "action" through $B$ can have any direction in the $n$-dimensional space $\mathbb{R}^{n}$.

The proof relies on a (new) global Carleman inequality that can be obtained for the solutions to the related adjoint systems

$$
\begin{cases}-\varphi_{t}-A^{*} \Delta \varphi=M(x, t)^{*} \varphi & \text { in } Q \\ \varphi=0 & \text { on } \Sigma \\ \varphi(x, T)=\varphi_{T}(x) & \text { in } \Omega\end{cases}
$$

where $\varphi_{T} \in L^{2}\left(\Omega ; \mathbb{R}^{n}\right)$; see Lemma 2.3. We have tried to explain that assumption (1.3) is necessary for this argument in Remark 2.4. At present, we have not been able to exclude it from the hypotheses.

Remark 1.2. Observe that assumptions (1.3) and (1.9) are sufficient to ensure the null controllability property of (1.1) for any diffusion and coupling matrices $A$ and $M=M(x, t)$. Assumption (1.9) is not necessary for proving the null controllability of system (1.1). Indeed, in $[6,27]$ the authors prove the (local) null controllability result of phase-field models by one control force $\left(n=2, n^{\prime}=1\right)$.

Remark 1.3. If $n^{\prime}<n$ and $M$ is a $L^{\infty}$ matrix-valued function, even when $A$ is a multiple of the identity, new phenomena can arise. More precisely, in [15] the authors prove that the approximate controllability property of a $2 \times 2$ linear system with $A=I$ depends on the position of the control set $\omega$. On the other hand, it is established in [10] that, in the same framework, the null controllability result holds when the control time $T>0$ is greater than a minimal time $T_{0}$ which depends on the coefficients of $M$. The null controllability result fails when $T<T_{0}$.

Now, let us introduce a locally Lipschitz-continuous function $f: \mathbb{R}^{n} \mapsto \mathbb{R}^{n}$ and let us consider the semilinear system

$$
\begin{cases}y_{t}-A \Delta y=f(y)+B v 1_{\omega} & \text { in } Q, \\ y=0 & \text { on } \Sigma, \\ y(x, 0)=y_{0}(x) & \text { in } \Omega .\end{cases}
$$


Again, we can speak of the null, exact to the trajectories or approximate controllability properties of (1.11). For instance, it will be said that (1.11) is exactly controllable to the trajectories at time $T$ if, for any $y_{0} \in$ $L^{2}\left(\Omega ; \mathbb{R}^{n}\right)$ and any weak solution $\widehat{y}$ corresponding to $v \equiv 0$, there exists a control $v \in L^{2}\left(\omega \times(0, T) ; \mathbb{R}^{n^{\prime}}\right)$ and an associated solution $y$ to (1.11) such that (1.5) holds. Now, this property is not equivalent but stronger than null controllability. On the other hand, it is not difficult to see that it also implies approximate controllability.

Our second main result in this paper is the following:

Theorem 1.4. Let $A$ and $B$ be as in Theorem 1.1. Asume that (1.2), (1.3) and (1.9) hold and

$$
f: \mathbb{R}^{n} \mapsto \mathbb{R}^{n} \text { is globally Lipschitz-continuous. }
$$

Then (1.11) is exactly controllable to the trajectories at any time T.

This result can be deduced from Theorem 1.1 (or, more precisely, from the Carleman inequality in Lem. 2.3 below) using arguments that are nowadays well known; see [23]. For completeness, we will provide the proof in Section 3. We will also see that the asumption (1.12) can be weakened so that, in particular, some slightly superlinear systems are controllable and, in fact, the action of the control can serve to avoid blow-up before $t=T$.

Let us come back to (1.1) and let us consider the particular case in which $M$ is constant. In this situation, it is possible to obtain controllability results also for $n^{\prime} \leq n$, provided $B$ satisfies appropriate conditions.

More precisely, let us denote by $\lambda_{1}, \lambda_{2}, \ldots$ the eigenvalues of the Dirichlet Laplacian in $\Omega$ and let us recall the notation (1.8). We then have the following theorem, which is the third main result in this paper:

Theorem 1.5. Let us assume that $A, M \in \mathcal{L}\left(\mathbb{R}^{n}\right), B \in \mathcal{L}\left(\mathbb{R}^{n^{\prime}} ; \mathbb{R}^{n}\right)$ and (1.2) and (1.3) hold. Then (1.1) is null controllable at time $T$ if and only if the following condition is satisfied:

$$
\operatorname{rank}\left[\lambda_{i} A-M \mid B\right]=n \quad \forall i \geq 1 .
$$

The same result was established in [5] in the case in which $A$ is diagonalizable. There, an observability inequality for a linear (adjoint) system is shown to be implied by a property satisfied by the solutions to a high order scalar PDE. The proof of Theorem 1.5 uses similar arguments; the details are given in Section 4 .

Notice that, in order to see whether or not (1.13) holds, one only has to check a finite amount of inequalities. This is because the $\lambda_{i}$ go to $+\infty$ as $i \rightarrow+\infty$ and, consequently, for $i$ large enough, they are outside the solution set of any algebraic equation of the form

$$
\operatorname{det} Z(\lambda)=0,
$$

where $Z(\lambda)$ is a minor of $\left[\lambda_{i} A-M \mid B\right]$.

An example of system that fulfills the assumptions of Theorem 1.5 is the following linearized two-phase solidification model, see [33,34]:

$$
\left\{\begin{array}{l}
\theta_{t}-\Delta \theta=\ell_{1} u_{t}+\ell_{2} w_{t}+m_{11} \theta+v 1_{\omega}, \\
u_{t}-\Delta u=\beta \theta+m_{22} u+m_{23} w \\
w_{t}-\Delta w=\beta \theta+m_{32} u+m_{33} w .
\end{array}\right.
$$

Here, we assume that $\beta$, the $\ell_{i}$ and the $m_{i j}$ are positive constants. The unknowns $\theta, u$ and $w$ can be interpreted as the temperature and two phase-field functions associated with two different kinds of solidification processes. It is not difficult to see that (1.14) can be written in the form (1.1), by replacing $u_{t}$ and $v_{t}$ in the first PDE. The result is:

$$
\left(\begin{array}{c}
\theta_{t} \\
u_{t} \\
w_{t}
\end{array}\right)-A\left(\begin{array}{c}
\Delta \theta \\
\Delta u \\
\Delta w
\end{array}\right)=M\left(\begin{array}{c}
\theta \\
u \\
w
\end{array}\right)+\left(\begin{array}{c}
v 1_{\omega} \\
0 \\
0
\end{array}\right),
$$


where

$$
A=\left(\begin{array}{ccc}
1 & \ell_{1} & \ell_{2} \\
0 & 1 & 0 \\
0 & 0 & 1
\end{array}\right), M=\left(\begin{array}{ccc}
m_{11}+\beta\left(\ell_{1}+\ell_{2}\right) & \ell_{1} m_{22}+\ell_{2} m_{32} & \ell_{1} m_{23}+\ell_{2} m_{33} \\
\beta & m_{22} & m_{23} \\
\beta & m_{32} & m_{33}
\end{array}\right) .
$$

A simple computation shows that the condition (1.13) in Theorem 1.5 is in this case independent of $\lambda_{i}$ and is satisfied if and only if

$$
m_{22}+m_{23} \neq m_{32}+m_{33} .
$$

In the sequel, $C, C_{0}, C_{1}, \ldots$ and $R$ are used to denote generic positive constants. Frequently, it will be convenient to specify the particular data on which they depend.

The rest of the paper is organized as follows.

In the next section, we present the proof of Theorem 1.1. As mentioned above, the main tool for this proof is a Carleman inequality for the solutions to (1.10). This is established by combining carefully appropriate Carleman estimates for similar scalar problems.

In Section 3, we give the proof of Theorem 1.4. As for similar scalar problems, this relies on a fixed-point argument. More precisely, we rewrite the controllability problem for (1.11) as a fixed-point equation for an adequate mapping. It will be seen that (1.12) (or some other assumption of this kind) is needed to bound uniformly the solutions, which justifies its inclusion in the result.

In Section 4, we give the proof of Theorem 1.5. As mentioned above, the main ideas for the proof have been adapted from $[4,5]$; the main estimates (again leading to appropriate observability inequalities) are established noting that the components of the solutions to the adjoint system solve a scalar PDE that is of the $n$-th order in $t$ and $-\Delta$.

Finally, Section 5 deals with some final comments and open questions.

\section{Proof of Theorem 1.1}

In the reminder of this paper, we will denote by $d_{\max }$ the maximal dimension of a Jordan block of the canonical form of $A$. By hypothesis, $d_{\max } \leq 4$.

The starting point is a basic global Carleman inequality for the solutions to scalar (real-valued and complexvalued) parabolic equations.

Thus, let $\alpha_{0}=\alpha_{0}(x)$ be a function satisfying

$$
\left\{\begin{array}{l}
\alpha_{0} \in C^{2}(\bar{\Omega}), \quad \alpha_{0}>0 \text { in } \Omega, \quad \alpha_{0}=0 \text { on } \partial \Omega, \\
\left|\nabla \alpha_{0}\right|>0 \text { in } \bar{\Omega} \backslash \omega .
\end{array}\right.
$$

Such a function exists, see [26]. Let us set

$$
\xi(x, t)=\frac{\mathrm{e}^{\lambda \alpha_{0}(x)}}{t(T-t)}, \alpha(x, t)=\frac{\mathrm{e}^{(\lambda+\mu)\left\|\alpha_{0}\right\|_{L} \infty}-\mathrm{e}^{\lambda \alpha_{0}(x)}}{t(T-t)}, \rho(x, t)=\mathrm{e}^{\alpha(x, t)},
$$

where $\lambda>0$ and $\mu>0$. The following notation will be used in order to abridge the estimates:

$$
\begin{aligned}
I_{m}(s, \lambda ; \psi):= & \iint_{Q} \rho^{-2 s}\left((s \xi)^{m-4}\left(\left|\psi_{t}\right|^{2}+|\Delta \psi|^{2}\right)\right. \\
& \left.+(s \xi)^{m-2} \lambda^{2}|\nabla \psi|^{2}+(s \xi)^{m} \lambda^{4}|\psi|^{2}\right)
\end{aligned}
$$

and

$$
I_{m, \omega}(s, \lambda ; \psi):=\iint_{\omega \times(0, T)} \rho^{-2 s}(s \xi)^{m} \lambda^{4}|\psi|^{2}
$$


for all $s, \lambda>0$, for any integer $m$ and for any sufficiently regular function $\psi=\psi(x, t)$ with values in $\mathbb{R}, \mathbb{C}$ or $\mathbb{R}^{n}$.

Let us consider the linear backwards in time scalar system

$$
\begin{cases}-\psi_{t}-\Delta \psi=g & \text { in } Q, \\ \psi=0 & \text { on } \Sigma, \\ \psi(x, T)=\psi_{T}(x) & \text { in } \Omega,\end{cases}
$$

where $g \in L^{2}(Q)$ and $\psi_{T} \in L^{2}(\Omega)$. In the following result, due to Fursikov and Imanuvilov [26], we recall the basic global Carleman estimates for the solutions to (2.3).

Lemma 2.1. For any integer $m$, there exist constants $s_{m}, \lambda_{m}$ and $C_{m}$ such that, for any $s \geq s_{m}$ and any $\lambda \geq \lambda_{m}$, the solutions to (2.3) satisfy

$$
I_{m}(s, \lambda ; \psi) \leq C_{m}\left(I_{m, \omega}(s, \lambda ; \psi)+\iint_{Q} \rho^{-2 s}(s \xi)^{m-3}|g|^{2}\right) .
$$

Furthermore, $\lambda_{m}$ and $C_{m}$ only depend on $m, \Omega$ and $\omega$ and $s_{m}$ can be taken of the form $s_{m}=\sigma_{m}\left(T+T^{2}\right)$, where $\sigma_{m}$ only depends on $m, \Omega$ and $\omega$.

For a detailed justification of the existence and properties of $\lambda_{m}, s_{m}$ and $C_{m}$, see the proof of Lemma 1.3 in $[24]$.

Secondly, let us consider the similar complex-valued system

$$
\begin{cases}-\psi_{t}-(a+i b) \Delta \psi=g & \text { in } Q, \\ \psi=0 & \text { on } \Sigma, \\ \psi(x, T)=\psi_{T}(x) & \text { in } \Omega,\end{cases}
$$

where now $a, b \in \mathbb{R}, a>0, g \in L^{2}(Q ; \mathbb{C})$ and $\psi_{T} \in L^{2}(\Omega ; \mathbb{C})$. It is also possible to deduce global Carleman estimates for the solutions to (2.5). They are given in the following lemma, whose proof is essentially given in $\mathrm{Fu}[25]$.

Lemma 2.2. For any integer $m$, there exist constants $s_{m}, \lambda_{m}$ and $C_{m}$ such that, for any $s \geq s_{m}$ and any $\lambda \geq \lambda_{m}$, the solutions to (2.5) satisfy (2.4). Furthermore, $\lambda_{m}$ and $C_{m}$ only depend on $m, \Omega, \omega, a$ and $b$ and $s_{m}$ can be taken of the form $s_{m}=\sigma_{m}\left(T+T^{2}\right)$, where $\sigma_{m}$ only depends on $m, \Omega, \omega, a$ and $b$.

Again, the existence and properties of $\lambda_{m}, s_{m}$ and $C_{m}$ are justified by the arguments in [24].

Let us set $g=g_{1}+i g_{2}$ and $\psi_{T}=\eta_{T}+i \zeta_{T}$ in (2.5). By writting the solutions in the form $\psi=\eta+i \zeta$, we see that they can also be regarded as solutions to the $2 \times 2$ system

$$
-\left(\begin{array}{l}
\eta_{t} \\
\zeta_{t}
\end{array}\right)-\left(\begin{array}{cc}
a & -b \\
b & a
\end{array}\right)\left(\begin{array}{c}
\Delta \eta \\
\Delta \zeta
\end{array}\right)=\left(\begin{array}{l}
g_{1} \\
g_{2}
\end{array}\right)
$$

together with Dirichlet boundary and initial conditions for $\eta$ and $\zeta$.

Let us denote by $M_{\infty}$ the norm of $M$ in $L^{\infty}\left(Q ; \mathcal{L}\left(\mathbb{R}^{n}\right)\right)$. Now, we present a Carleman estimate for the solutions to the non-scalar (adjoint) problem (1.10):

Lemma 2.3. Let the assumptions in Theorem 1.1 be satisfied. For any integer $m$, there exist constants $s_{m}^{\prime}, \lambda_{m}^{\prime}$ and $C_{m}^{\prime}$ such that, for any $s \geq s_{m}^{\prime}$ and any $\lambda \geq \lambda_{m}^{\prime}$, the solutions to (1.10) satisfy

$$
I_{m-3}(s, \lambda ; \varphi) \leq C_{m}^{\prime} I_{m, \omega}(s, \lambda ; \varphi) .
$$

Furthermore, $C_{m}^{\prime}$ only depends on $m, \Omega, \omega$ and $A, \lambda_{m}^{\prime}$ only depends on $m, \Omega, \omega, A$ and $M_{\infty}$ and $s_{m}^{\prime}$ can be taken of the form $s_{m}^{\prime}=\sigma_{m}^{\prime}\left(T+T^{2}\right)$, where $\sigma_{m}^{\prime}$ only depends on $m, \Omega, \omega, A$ and $M_{\infty}$. 
Proof. In this proof, we will denote by $C_{0}$ a generic positive constant only depending on $\Omega, \omega$ and $A$.

First, notice that it can be assumed that $A$ is written in the canonical form. Indeed, there exists a non-singular matrix $P \in \mathcal{L}\left(\mathbb{C}^{n}\right)$ such that $A=P J P^{-1}$ for some $J \in \mathcal{L}\left(\mathbb{C}^{n}\right)$ of the form

$$
J=\operatorname{diag}\left(J_{1}, \ldots, J_{s}\right),
$$

where the $J_{i}$ are the Jordan blocks associated to the eigenvalues $\mu_{i}$ of $A$. By hypothesis, we have (1.2) and this implies that, for all $i$,

$$
J_{i}=\left[\begin{array}{cccc}
\mu_{i} & 1 & & \\
& \mu_{i} & 1 & \\
& & \mu_{i} & 1 \\
& & & \mu_{i}
\end{array}\right]
$$

or a matrix with the same shape and smaller dimension, with $\operatorname{Re} \mu_{i}>0$.

The solutions to (1.10) can be put in correspondance with the solutions to

$$
\begin{cases}-\psi_{t}-J^{*} \Delta \psi=P^{*} M(x, t)^{*}\left(P^{*}\right)^{-1} \psi & \text { in } Q, \\ \psi=0 & \text { on } \Sigma, \\ \psi(x, T)=P^{*} \varphi_{T}(x) & \text { in } \Omega,\end{cases}
$$

through the change of variable $\varphi=\left(P^{*}\right)^{-1} \psi$ and, obviously, it suffices to prove (2.6) for $\psi$.

For instance, let us assume that, in (2.8), the first four PDEs correspond to the same block and let us write them in the form

$$
\begin{aligned}
& -\psi_{1, t}-\bar{\mu} \Delta \psi_{1}=\sum_{j=1}^{n} \tilde{M}_{1 j}(x, t) \psi_{j}, \\
& -\psi_{2, t}-\bar{\mu} \Delta \psi_{2}=\sum_{j=1}^{n} \tilde{M}_{2 j}(x, t) \psi_{j}+\Delta \psi_{1}, \\
& -\psi_{3, t}-\bar{\mu} \Delta \psi_{3}=\sum_{j=1}^{n} \tilde{M}_{3 j}(x, t) \psi_{j}+\Delta \psi_{2}, \\
& -\psi_{4, t}-\bar{\mu} \Delta \psi_{4}=\sum_{j=1}^{n} \tilde{M}_{4 j}(x, t) \psi_{j}+\Delta \psi_{3},
\end{aligned}
$$

where the $\tilde{M}_{i j}(x, t)$ stand for the components of the matrix $P^{*} M(x, t)^{*}\left(P^{*}\right)^{-1}$.

Let us write (2.4) for $\psi_{1}, \psi_{2}, \psi_{3}$ and $\psi_{4}$ respectively with $m=3,2,1$ and 0 . The following is found for all large $s$ and $\lambda$ :

$$
\begin{aligned}
& I_{3}\left(s, \lambda ; \psi_{1}\right) \leq C_{0}\left(I_{3, \omega}\left(s, \lambda ; \psi_{1}\right)+M_{\infty}^{2} \iint_{Q} \rho^{-2 s}|\psi|^{2}\right), \\
& I_{2}\left(s, \lambda ; \psi_{2}\right) \leq C_{0}\left(I_{2, \omega}\left(s, \lambda ; \psi_{2}\right)+M_{\infty}^{2} \iint_{Q} \rho^{-2 s}(s \xi)^{-1}|\psi|^{2}+\iint_{Q} \rho^{-2 s}(s \xi)^{-1}\left|\Delta \psi_{1}\right|^{2}\right), \\
& I_{1}\left(s, \lambda ; \psi_{3}\right) \leq C_{0}\left(I_{1, \omega}\left(s, \lambda ; \psi_{3}\right)+M_{\infty}^{2} \iint_{Q} \rho^{-2 s}(s \xi)^{-2}|\psi|^{2}+\iint_{Q} \rho^{-2 s}(s \xi)^{-2}\left|\Delta \psi_{2}\right|^{2}\right), \\
& I_{0}\left(s, \lambda ; \psi_{4}\right) \leq C_{0}\left(I_{0, \omega}\left(s, \lambda ; \psi_{4}\right)+M_{\infty}^{2} \iint_{Q} \rho^{-2 s}(s \xi)^{-3}|\psi|^{2}+\iint_{Q} \rho^{-2 s}(s \xi)^{-3}\left|\Delta \psi_{3}\right|^{2}\right) .
\end{aligned}
$$


Therefore, an appropriate linear combination of the left hand sides can be used to control and absorb all the second-order terms in the right. Indeed, it is clear that $(s \xi)^{n_{1}} \leq C(s \xi)^{n_{2}}$ whenever $n_{1} \leq n_{2}$. Accordingly, if $s \geq \sigma_{0}\left(T+T^{2}\right)$ for some $\sigma_{0}$ only depending on $\Omega$ and $\omega$, we have:

$$
\begin{aligned}
& I_{3}\left(s, \lambda ; \psi_{1}\right)+I_{2}\left(s, \lambda ; \psi_{2}\right)+I_{1}\left(s, \lambda ; \psi_{3}\right)+I_{0}\left(s, \lambda ; \psi_{4}\right) \\
& \quad \leq C_{0}\left(I_{3, \omega}\left(s, \lambda ; \psi_{1}\right)+I_{2, \omega}\left(s, \lambda ; \psi_{2}\right)+I_{1, \omega}\left(s, \lambda ; \psi_{3}\right)+I_{0, \omega}\left(s, \lambda ; \psi_{4}\right)\right)+C_{0} M_{\infty}^{2} \iint_{Q} \rho^{-2 s}|\psi|^{2} .
\end{aligned}
$$

In order to absorb as many terms as possible in the last integral in the right hand side, we do as follows:

(1) We take $s \geq s_{3}+C_{0} M_{\infty}^{2 / 3} T^{2}$ and $\lambda \geq \lambda_{3}$; this makes it possible to skip $\left|\psi_{1}\right|^{2}$.

(2) Then, we take $s$ and $\lambda$ as before and also satisfying $s \geq s_{2}+C_{0} M_{\infty} T^{2}$ and $\lambda \geq \lambda_{2}$; in this way, we can suppress $\left|\psi_{2}\right|^{2}$.

(3) Then, with $s$ and $\lambda$ as in the previous step and also satisfying $s \geq s_{1}+C_{0} M_{\infty}^{2} T^{2}$ and $\lambda \geq \lambda_{2}$, we can also skip $\left|\psi_{3}\right|^{2}$ and, finally,

(4) We choose $s$ and $\lambda$ as in the previous step and also satisfying $s \geq s_{0}$ and $\lambda \geq \lambda_{0}+C_{0} M_{\infty}^{1 / 2}$, in order to skip $\left|\psi_{4}\right|^{2}$.

Hence, there exist $s_{3}^{\prime}$ and $\lambda_{3}^{\prime}$ (as in the statement) such that, for all $s \geq s_{3}^{\prime}$ and $\lambda \geq \lambda_{3}^{\prime}$, one has:

$$
\begin{aligned}
& I_{3}\left(s, \lambda ; \psi_{1}\right)+I_{2}\left(s, \lambda ; \psi_{2}\right)+I_{1}\left(s, \lambda ; \psi_{3}\right)+I_{0}\left(s, \lambda ; \psi_{4}\right) \\
& \quad \leq C_{0}\left(I_{3, \omega}\left(s, \lambda ; \psi_{1}\right)+I_{2, \omega}\left(s, \lambda ; \psi_{2}\right)+I_{1, \omega}\left(s, \lambda ; \psi_{3}\right)+I_{0, \omega}\left(s, \lambda ; \psi_{4}\right)\right)+C_{0} M_{\infty}^{2} \iint_{Q} \rho^{-2 s} \sum_{j \geq 5}\left|\psi_{j}\right|^{2} .
\end{aligned}
$$

Obviously, similar estimates can also be obtained for the $\psi_{i}$ corresponding to any other Jordan block of equal or lower dimension.

It is also clear that, if we choose $s$ and eventually $\lambda$ as indicated, after addition, we get in the left hand side terms that can absorb all the zero-order terms in the right. Therefore,

$$
I_{0}(s, \lambda ; \psi) \leq C_{0} I_{3, \omega}(s, \lambda ; \psi)
$$

for all $s \geq s_{3}^{\prime}, \lambda \geq \lambda_{3}^{\prime}$. This proves the lemma for $m=3$.

With similar computations, it is possible to prove (2.6) for any other integer $m$. We skip the details, that can be easily deduced from the previous argument.

Remark 2.4. From the proof of this lemma, we see that the best possible choices of $s_{m}^{\prime}$ and $\lambda_{m}^{\prime}$ are as follows (recall that $d_{\max }$ denotes the maximal dimension of a Jordan block for $A$ ):

- If $d_{\max }=1$ (i.e. $A$ is diagonalizable), then we can choose $s_{m}^{\prime}=s_{m}+C_{0} M_{\infty}^{2 / 3} T^{2}$ and $\lambda_{m}^{\prime}=\lambda_{m}$.

- If $d_{\max }=2$, we can take $s_{m}^{\prime}=s_{m}+C_{0} M_{\infty} T^{2}$ and $\lambda_{m}^{\prime}=\lambda_{m}$.

- If $d_{\max }=3$, then we need $s_{m}^{\prime}=s_{m}+C_{0} M_{\infty}^{2} T^{2}$ and $\lambda_{m}^{\prime}=\lambda_{m}$.

- Finally, if $d_{\max }=4$, we have to take $s_{m}^{\prime}=s_{m}$ and $\lambda_{m}^{\prime}=\lambda_{m}+C_{0} M_{\infty}^{2}$.

Remark 2.5. It is also clear that a proof of (2.6) of the same kind cannot work when $d_{\max } \geq 5$. Indeed, if (for instance) the first block is of dimension 5 , the associated components $\psi_{1}, \ldots, \psi_{5}$ are coupled through second-order terms and we must add to (2.9) a fifth PDE:

$$
-\psi_{5, t}-\bar{\mu} \Delta \psi_{5}=\sum_{j=1}^{n} \tilde{M}_{5 j}(x, t) \psi_{j}+\Delta \psi_{4} .
$$


If we look for an estimate of $\psi_{5}$ and we try to incorporate a new Carleman inequality to (2.10), in view of the term $\Delta \psi_{4}$ in the right hand side, we are forced to take $m=-1$. But then the zero-order term for $\psi_{5}$ that we obtain in the left is

$$
\iint_{Q} \rho^{-2 s}(s \xi)^{-1} \lambda^{4}\left|\psi_{5}\right|^{2}
$$

and this is not sufficient to control the similar zero-order term in the right coming from the first Carleman inequality in (2.10).

We can now achieve the proof of Theorem 1.1.

In the remainder of this section, $R$ (resp. $C$ ) denotes various positive constants only depending on $\Omega, \omega, A$ and $M_{\infty}$ (resp. $\Omega, \omega, A, M_{\infty}$ and $\left.T\right)$.

First, recall that, in view of classical arguments, the null controllabilty of (1.1) is equivalent to the observability of (1.10), that is, to the estimate

$$
\|\varphi(\cdot, 0)\|_{L^{2}}^{2} \leq C \iint_{\omega \times(0, T)}\left|B^{*} \varphi\right|^{2}
$$

for any solution to (1.10); for a detailed explanation, see for instance [24].

In view of the assumption (1.9), this is also equivalent to the simpler estimate

$$
\|\varphi(\cdot, 0)\|_{L^{2}}^{2} \leq C \iint_{\omega \times(0, T)}|\varphi|^{2} .
$$

Therefore, let us check that the Carleman inequality (2.6), together with the usual parabolic energy estimates, imply (2.13) for some $C$.

Indeed, let us take (for example) $m=3, s=s_{3}^{\prime}$ and $\lambda=\lambda_{3}^{\prime}$ in (2.6). In view of the energy estimates

$$
-\frac{\mathrm{d}}{\mathrm{d} t}\|\varphi\|_{L^{2}}^{2}+2 a_{0}\|\nabla \varphi\|_{L^{2}}^{2} \leq R\|\varphi\|_{L^{2}}^{2},
$$

we find that

$$
\|\varphi(\cdot, 0)\|_{L^{2}}^{2} \leq \frac{2}{T} \mathrm{e}^{R T} \int_{T / 4}^{3 T / 4}\|\varphi(\cdot, t)\|_{L^{2}}^{2} \mathrm{~d} t .
$$

The left hand side of (2.6) is bounded from below as follows:

$$
\begin{aligned}
I_{0}\left(s_{3}^{\prime}, \lambda_{3}^{\prime} ; \varphi\right) & \geq \iint_{Q} \rho^{-2 s_{3}^{\prime}} \lambda_{3}^{\prime 4}|\varphi|^{2} \\
& \geq R \int_{T / 4}^{3 T / 4} \mathrm{e}^{-2 s_{3}^{\prime} \alpha^{*}(t)}\|\varphi(\cdot, t)\|_{L^{2}}^{2} \mathrm{~d} t
\end{aligned}
$$

where

$$
\alpha^{*}(t):=\max _{\bar{\Omega}} \alpha(x, t) \leq \frac{R}{T^{2}} .
$$

Since $s_{3}^{\prime}$ has the form $s_{3}^{\prime}=R\left(T+T^{2}\right)$, the following is obtained:

$$
I_{0}\left(s_{3}^{\prime}, \lambda_{3}^{\prime} ; \varphi\right) \geq \mathrm{e}^{-R\left(1+\frac{1}{T}\right)} \int_{T / 4}^{3 T / 4}\|\varphi(\cdot, t)\|_{L^{2}}^{2} \mathrm{~d} t
$$

and this inequality, together with (2.14), yields:

$$
I_{0}\left(s_{3}^{\prime}, \lambda_{3}^{\prime} ; \varphi\right) \geq \mathrm{e}^{-R\left(1+T+\frac{1}{T}\right)}\|\varphi(\cdot, 0)\|_{L^{2}}^{2} .
$$


On the other hand, the right hand of (2.6) can be easily bounded from above:

$$
\begin{aligned}
I_{3, \omega}\left(s_{3}^{\prime}, \lambda_{3}^{\prime} ; \varphi\right) & =\iint_{\omega \times(0, T)} \rho^{-2 s_{3}^{\prime}\left(s_{3}^{\prime} \xi\right)^{3} \lambda_{3}^{\prime 4}|\varphi|^{2}} \\
& \leq R \iint_{\omega \times(0, T)} \frac{1}{t^{3}(T-t)^{3}} \mathrm{e}^{-\frac{R\left(T+T^{2}\right)}{t(T-t)}|\varphi|^{2}} \\
& \leq \iint_{\omega \times(0, T)} \mathrm{e}^{-R\left(1+\frac{1}{T}\right)|\varphi|^{2}}
\end{aligned}
$$

and, from (2.15) and (2.16), we find (2.13) with a constant $C$ of the form

$$
C=\mathrm{e}^{R\left(1+T+\frac{1}{T}\right)}
$$

Consequently, (1.10) is observable and the null controllability of (1.1) is proved.

Remark 2.6. From (2.13) and the fact $C$ takes the form (2.17), we find an estimate of the cost $\mathcal{C}\left(y_{0}\right)$ of the null controllability of (1.1). In other words, one has:

$$
\begin{aligned}
\mathcal{C}\left(y_{0}\right) & :=\inf \left\{\|v\|_{L^{2}\left(\omega \times(0, T) ; \mathbb{R}^{n^{\prime}}\right)}: y \text { and } v \text { satisfy (1.1) and (1.4) }\right\} \\
& \leq \mathrm{e}^{K\left(1+T+\frac{1}{T}\right)}\left\|y_{0}\right\|_{L^{2}} .
\end{aligned}
$$

In fact, this estimate can be improved if we use the "optimal" $s_{m}^{\prime}$ and $\lambda_{m}^{\prime}$ indicated in Remark 2.4. Thus, it can be seen that $\mathcal{C}\left(y_{0}\right) \leq C_{*}\left\|y_{0}\right\|_{L^{2}}$, where

$$
C_{*}= \begin{cases}\mathrm{e}^{R\left(1+\frac{1}{T}+M_{\infty}^{2 / 3}\right)} & \text { if } d_{\max }=1 \\ \mathrm{e}^{R\left(1+\frac{1}{T}+M_{\infty}\right)} & \text { if } d_{\max }=2, \\ \mathrm{e}^{R\left(1+\frac{1}{T}+M_{\infty}^{2}\right)} & \text { if } d_{\max }=3 \\ \mathrm{e}^{R\left(1+\frac{1}{T}+\mathrm{e}^{R M_{\infty}^{2}}\right)} & \text { if } d_{\max }=4\end{cases}
$$

and $R$ only depends on $\Omega, \omega$ and $A$. The computations are lenghty and will be omitted.

Remark 2.7. Similar results to Theorem 1.1 can be established for linear systems of the form

$$
\begin{cases}y_{t}-A \Delta y=M(x, t) y+\sum_{k=1}^{N} \partial_{k}\left(W^{k}(x, t) y\right)+B v 1_{\omega} & \text { in } Q \\ y=0 & \text { on } \Sigma \\ y(x, 0)=y_{0}(x) & \text { in } \Omega\end{cases}
$$

and

$$
\begin{cases}y_{t}-A \Delta y=M(x, t) y+\sum_{k=1}^{N} W^{k}(x, t) \partial_{k} y+B v 1_{\omega} & \text { in } Q \\ y=0 & \text { on } \Sigma \\ y(x, 0)=y_{0}(x) & \text { in } \Omega\end{cases}
$$

with $M, W^{k} \in L^{\infty}\left(Q ; \mathcal{L}\left(\mathbb{R}^{n}\right)\right)$. In these cases, in order to prove the observability of the associated adjoint states, we have to assume that the Jordan blocks of the canonical form of $A$ must have dimensions $\leq 2$. Indeed, let us see this for instance for (2.19). The adjoint system is the following one:

$$
\begin{cases}-\varphi_{t}-A^{*} \Delta \varphi=M(x, t)^{*} \varphi-\sum_{k=1}^{N} W^{k}(x, t)^{*} \partial_{k} \varphi & \text { in } Q \\ \varphi=0 & \text { on } \Sigma \\ \varphi(x, T)=\varphi_{T}(x) & \text { in } \Omega .\end{cases}
$$


Again, it can be assumed that $A$ is in the canonical form. In the Carleman estimates corresponding to the components $\varphi_{1}, \varphi_{2}, \ldots$ associated to a Jordan block, we find in the left hand side exponents of $s$ of orders 1,0 , -1 , etc. for $\left|\nabla \varphi_{1}\right|^{2},\left|\nabla \varphi_{2}\right|^{2},\left|\nabla \varphi_{3}\right|^{2}$, etc. However, in the right hand side, we now have all the $\left|\nabla \varphi_{i}\right|^{2}$ multiplied by $s^{0}$. Consequently, we can deal with Jordan blocks of at most dimension 2 .

\section{Proof of Theorem 1.4}

This section is devoted to proving that, under the assumptions in Theorem 1.4, the semilinear system (1.11) is exactly controllable to the trajectories. The proof of this controllability property can be obtained from Lemma 2.3 essentially as in the scalar case; see $[23,26]$.

The argument is the following.

Let us fix a trajectory $\widehat{y}$, that is, a weak solution to (1.11) for $v \equiv 0$. By introducing the change of variable $y=\widehat{y}+w$, it is clear that what we have to prove is the null controllability of the system

$$
\begin{cases}w_{t}-A \Delta w=h(x, t ; w)+v 1_{\omega} & \text { in } Q \\ w=0 & \text { on } \Sigma \\ w(x, 0)=w_{0}(x) & \text { in } \Omega\end{cases}
$$

where we have introduced

$$
h(x, t ; s):=f(s+\widehat{y}(x, t))-f(\widehat{y}(x, t)) .
$$

Let us first assume that $f \in C^{1}\left(\mathbb{R}^{n} ; \mathbb{R}^{n}\right)$ (and is globally Lipschitz).

Notice that, for each $i, h_{i}(x, t ; w)$ can be written in the form

$$
h_{i}(x, t ; w)=\sum_{\ell=1}^{n}\left(\int_{0}^{1} \partial_{\ell} f_{i}(\sigma w+\widehat{y}(x, t)) d \sigma\right) w_{\ell}:=G_{i}(x, t ; w) w
$$

and then

$$
h(x, t ; w) \equiv G(x, t ; w) w \text { for some } G \in C^{0}\left(Q \times \mathbb{R}^{n} ; \mathcal{L}\left(\mathbb{R}^{n}\right)\right) .
$$

Consequently, it suffices to find a fixed-point of the mapping $z \mapsto w$, where we assume that $w$ is, together with some $v \in L^{2}\left(\omega \times(0, T) ; \mathbb{R}^{n}\right)$, a solution to the linear system

$$
\begin{cases}w_{t}-A \Delta w=G(x, t ; z) w+v 1_{\omega} & \text { in } Q \\ w=0 & \text { on } \Sigma \\ w(x, 0)=w_{0}(x) & \text { in } \Omega\end{cases}
$$

satisfying

$$
w(x, T)=0 \text { in } \Omega .
$$

Observe that $G=\left\{G_{i j}\right\}$ for some uniformly bounded Carathéodory functions $G_{i j}: Q \times \mathbb{R}^{n} \mapsto \mathbb{R}$. Let us denote by $G_{\infty}$ the norm of $G$ in $L^{\infty}\left(Q \times \mathbb{R}^{n} ; \mathcal{L}\left(\mathbb{R}^{n}\right)\right)$. Then (3.2) is a system of the form (1.1) and, for each $z \in L^{2}\left(Q ; \mathbb{R}^{n}\right)$, we can apply Theorem 1.1 to $(3.2)$ and deduce that there exist controls $v \in L^{2}\left(\omega \times(0, T) ; \mathbb{R}^{n}\right)$ and associated states $w$ satisfying (3.3). It is also clear from this theorem and Remark 2.6 that $v$ and $w$ can be found satisfying the following estimates:

$$
\|v\|_{L^{2}\left(\omega \times(0, T) ; \mathbb{R}^{n}\right)} \leq \mathrm{e}^{R\left(1+T+\frac{1}{T}\right)}\left\|w_{0}\right\|_{L^{2}}
$$

and

$$
\|w\|_{L^{2}\left(Q ; \mathbb{R}^{n}\right)} \leq \mathrm{e}^{R\left(1+T+\frac{1}{T}\right)}\left\|w_{0}\right\|_{L^{2}}
$$

where $R$ depends on $\Omega, \omega, A$ and $G_{\infty}$ but is independent of $z \in L^{2}\left(Q ; \mathbb{R}^{n}\right)$. 
Let us introduce the multi-valued mappings $\Lambda_{0}: L^{2}\left(Q ; \mathbb{R}^{n}\right) \mapsto L^{2}\left(\omega \times(0, T) ; \mathbb{R}^{n}\right)$ and $\Lambda: L^{2}\left(Q ; \mathbb{R}^{n}\right) \mapsto$ $L^{2}\left(Q ; \mathbb{R}^{n}\right)$, with

$$
\Lambda_{0}(z)=\left\{v \in L^{2}\left(\omega \times(0, T) ; \mathbb{R}^{n}\right): v \text { is a null control for (3.2) satisfying (3.4) }\right\}
$$

and

for all $z \in L^{2}\left(Q ; \mathbb{R}^{n}\right)$.

$$
\Lambda(z)=\left\{w \in L^{2}\left(Q ; \mathbb{R}^{n}\right): w \text { solves }(3.2) \text { with } v \in \Lambda_{0}(z)\right\}
$$

At this point, it is easy to check that, as in the scalar case, $\Lambda$ satisfies all the hypotheses of Kakutani's fixed-point Theorem (see for instance [12]). Consequently, $\Lambda$ possesses at least one fixed-point $w \in L^{2}\left(Q ; \mathbb{R}^{n}\right)$ and, obviously, $w$ solves together with some $v \in L^{2}\left(\omega \times(0, T) ; \mathbb{R}^{n}\right)$ the nonlinear system (3.1) and satisfies (3.3). This proves Theorem 1.4 when $f \in C^{1}\left(\mathbb{R}^{n} ; \mathbb{R}^{n}\right)$.

Let us now assume that $f$ satisfies (1.12) but is not necessarily $C^{1}$. Then, there exist regular functions $f_{1}, f_{2}, \ldots$ satisfying

$$
\begin{aligned}
& f_{k} \rightarrow f \text { weakly- } * \text { in } W^{1, \infty}\left(\mathbb{R}^{n} ; \mathbb{R}^{n}\right), \text { strongly in } L_{\text {loc }}^{2}\left(\mathbb{R}^{n} ; \mathbb{R}^{n}\right) \text { and a.e. } \\
& \left|f_{k}^{\prime}\right| \leq C \text { in } \mathbb{R}^{n} \forall k \geq 1 .
\end{aligned}
$$

We can solve the nonlinear null controllability problems associated to the $f_{k}$ and find controls $v_{k}$ and states $w_{k}$ uniformly bounded in the $L^{2}$ norms. After some work, using standard energy estimates, we can extract convergent sequences and deduce that the limits $v$ and $w$ again satisfy (3.1) and (3.3).

This ends the proof of Theorem 1.4.

Remark 3.1. Kakutani's Theorem has been used in the context of the controllability of nonlinear PDEs a lot of times. See for instance [18,21,23].

Remark 3.2. As we said in Section 1, the hypothesis (1.12) can be weakened. In fact, a more involved fixedpoint argument shows that (1.11) is exactly controllable to the trajectories whenever the following conditions are satisfied:

(1) $A$ and $B$ are as in Theorem 1.1.

(2) $f: \mathbb{R}^{n} \mapsto \mathbb{R}^{n}$ is locally Lipschitz-continuous and there exists at least one globally defined solution $\bar{y}=\bar{y}(x, t)$ to the PDE in (1.11), with

$$
\bar{y} \in L^{\infty}\left(Q ; \mathbb{R}^{n}\right) \cap L^{2}\left(0, T ; H_{0}^{1}\left(\Omega ; \mathbb{R}^{n}\right)\right)
$$

(3) One has

$$
\limsup _{|z| \rightarrow+\infty} \frac{|f(z)|}{|z| X(|z|)}=0
$$

where the function $X: \mathbb{R}_{+} \mapsto \mathbb{R}_{+}$is given by

$$
X(r)= \begin{cases}(\log (1+r))^{3 / 2} & \text { if } d_{\max }=1 \\ (\log (1+r)) & \text { if } d_{\max }=2 \\ (\log (1+r))^{1 / 2} & \text { if } d_{\max }=3 \\ (\log (\log (1+r))) & \text { if } d_{\max }=4\end{cases}
$$

To get this result, it suffices to prove that, under these assumptions, when $y_{0} \in L^{\infty}\left(\Omega ; \mathbb{R}^{n}\right)$, the multi-valued mapping $\Lambda$ possesses at least one fixed-point in $L^{\infty}\left(Q ; \mathbb{R}^{n}\right)$. This can be shown arguing as in the scalar case; the main tool is an improved obervability estimate of the form

$$
\|\varphi(\cdot, 0)\|_{L^{2}}^{2} \leq C\left(\iint_{\omega \times(0, T)}|\varphi|\right)^{2},
$$

where $C$ behaves similarly to the constant $C_{*}$ in (2.18). We omit the details, that can again be deduced easily adapting the arguments in [23]. 


\section{Proof of Theorem 1.5}

In this section, we will assume that $M$ is a constant matrix.

Recall that the null controllability of (1.1) is equivalent to the existence of a constant $C$ such that

$$
\|\varphi(\cdot, 0)\|_{L^{2}}^{2} \leq C \iint_{\omega \times(0, T)}\left|B^{*} \varphi\right|^{2}
$$

for any solution to (1.10).

Let us first assume that (1.1) is null-controllable.

If we have $\operatorname{rank}\left[\lambda_{i} A-M \mid B\right] \leq n-1$ for some $i$, then it is very easy to check that the associated ordinary differential system is not null-controllable. In other words, there exists $\Phi_{T} \in \mathbb{R}^{n} \backslash\{0\}$ such that the solution to the Cauchy problem

$$
-\Phi_{t}+\left(\lambda_{i} A^{*}-M^{*}\right) \Phi=0, \quad t \in(0, T) ; \quad \Phi(T)=\Phi_{T}
$$

satisfies

$$
B^{*} \Phi(t) \equiv 0
$$

If we now set $\varphi_{T}=\Phi_{T} w^{i}$, where $w^{i}$ is an eigenfunction associated to $\lambda_{i}$, we see that the corresponding solution to (1.10) cannot satisfy (4.1).

Consequently, (1.13) must hold.

Conversely, let us assume that (1.13) is satisfied. In order to prove that (1.1) is null-controllable, we will adapt some arguments from [5]. More precisely, the following estimates will be established:

(1) An estimate of $\|\varphi(\cdot, t)\|_{L^{2}}$ in terms of an integral in $\Omega$ concerning high-order spatial derivatives of $\varphi$ :

$$
\int_{\Omega}|\varphi(x, t)|^{2} \mathrm{~d} x \leq R \int_{\Omega}\left|(-\Delta)^{k}\left(K_{*} \varphi\right)(x, t)\right|^{2} \mathrm{~d} x
$$

for any $t \in[0, T)$ and any $k \geq 2(n-1)^{2}$, where $R$ only depends on $n, A$ and $M$. Here, the components of $K_{*} \varphi$ are appropriate linear combinations of the components of $\varphi$ and their second-order in space derivatives; see (4.4) below.

Notice that, for all $t \in[0, T), \varphi(\cdot, t)$ is regular enough to give a sense to $(-\Delta)^{k}\left(K_{*} \varphi\right)(\cdot, t)$, which belongs to $L^{2}(\Omega)$.

(2) A weighted global estimate of these high-order derivatives in terms of a local integral of $\left|B^{*} \varphi\right|^{2}$ :

$$
\iint_{Q} \hat{\rho}^{-2 s}\left|(-\Delta)^{k}\left(K_{*} \varphi\right)\right|^{2} \leq C \iint_{\omega \times(0, T)}\left|B^{*} \varphi\right|^{2}
$$

for some $\hat{\rho}=\hat{\rho}(t)$.

From (4.2) and (4.3), we will easily deduce (4.1) and, therefore, the null controllability of (1.1).

\subsection{Proof of the space-like estimate (4.2)}

Let us introduce the operators $K: D(K) \subset L^{2}\left(\Omega ; \mathbb{R}^{n n^{\prime}}\right) \mapsto L^{2}\left(\Omega ; \mathbb{R}^{n}\right)$ and $K_{*}: D\left(K_{*}\right) \subset L^{2}\left(\Omega ; \mathbb{R}^{n}\right) \mapsto$ $L^{2}\left(\Omega ; \mathbb{R}^{n n^{\prime}}\right)$, with

$$
\begin{aligned}
D(K) & =\left\{v \in L^{2}\left(\Omega ; \mathbb{R}^{n n^{\prime}}\right):[-A \Delta-M \mid B] v \in L^{2}\left(\Omega ; \mathbb{R}^{n}\right)\right\} \\
D\left(K_{*}\right) & =\left\{\varphi \in L^{2}\left(\Omega ; \mathbb{R}^{n}\right):[-A \Delta-M \mid B]^{*} \varphi \in L^{2}\left(\Omega ; \mathbb{R}^{n n^{\prime}}\right)\right\}
\end{aligned}
$$

and

$$
K v:=[-A \Delta-M \mid B] v, \quad K_{*} \varphi:=[-A \Delta-M \mid B]^{*} \varphi .
$$

Then $K$ and $K_{*}$ are densely defined unbounded linear operators. 
Let us also introduce the matrices $K_{i}$, with

$$
K_{i}:=\left[\lambda_{i} A-M \mid B\right] \in \mathcal{L}\left(\mathbb{R}^{n n^{\prime}} ; \mathbb{R}^{n}\right) \quad \forall i \geq 1 .
$$

In order to prove (4.2), we first notice that the hypothesis (1.13) can be recast in the form

$$
\operatorname{det} K_{i} K_{i}^{*} \neq 0 \quad \forall i \geq 1
$$

Let $f \in L^{2}\left(\Omega ; \mathbb{R}^{n}\right)$ be given. We have

$$
f=\sum_{i \geq 1} a^{i} w^{i}
$$

for some $a^{i} \in \mathbb{R}^{n}$. Let us assume for the moment that $a^{i}=0$ for all $i \geq p+1$, for some $p \geq 1$. Then $K_{*} f$ and $(-\Delta)^{k} K_{*} f$ are well-defined and belong to $L^{2}\left(\Omega ; \mathbb{R}^{n n^{\prime}}\right)$ and one has

$$
K_{*} f=\sum_{i}\left(K_{i}^{*} a^{i}\right) w^{i}, \quad(-\Delta)^{k} K_{*} f=\sum_{i} \lambda_{i}^{k}\left(K_{i}^{*} a^{i}\right) w^{i}
$$

whence

$$
\left\|(-\Delta)^{k} K_{*} f\right\|_{L^{2}}^{2}=\sum_{i} \lambda_{i}^{2 k}\left|K_{i}^{*} a^{i}\right|^{2}
$$

Let us denote by $\kappa_{j}^{i}$, for $1 \leq j \leq n$, the (real and nonnegative) eigenvalues of $K_{i} K_{i}^{*}$. Then, we have the following for all $i$ :

$$
\left|K_{i}^{*} a^{i}\right|^{2}=\left(K_{i} K_{i}^{*} a^{i}, a^{i}\right) \geq \kappa_{1}^{i}\left|a^{i}\right|^{2} .
$$

On the other hand, there exists $C_{1}$ (independent of $i$ ) such that

$$
\operatorname{det} K_{i} K_{i}^{*} \geq C_{1} \quad \forall i \geq 1 .
$$

Indeed, let us set $\tilde{k}(\lambda):=\operatorname{det} \tilde{K}(\lambda) \tilde{K}(\lambda)^{*}$ for all $\lambda$, where

$$
\tilde{K}(\lambda):=[\lambda A-M \mid B] .
$$

Then, $\tilde{k}$ is a polynomial function of degree $2 n(n-1), \tilde{k}(\lambda) \geq 0$ for all $\lambda$ and, by assumption, $\tilde{k}\left(\lambda_{i}\right) \neq 0$ for all $i$. All possible solutions to the equation $\tilde{k}(\lambda)=0$ satisfy $|\lambda|<R$ for some $R>0$ and there exists $C_{2}>0$ such that $\tilde{k}(\lambda) \geq C_{2}$ for $|\lambda| \geq R$. Moreover, for some $\ell$, one has $\lambda_{\ell}>R$. Hence,

- Either $i \leq \ell-1$ and then

$$
\operatorname{det} K_{i} K_{i}^{*} \geq C_{3}:=\min _{j \leq \ell-1}\left(\operatorname{det} K_{j} K_{j}^{*}\right)
$$

- Or $i \geq \ell$ and then $\lambda_{i} \geq \lambda_{\ell}>R$ and

$$
\operatorname{det} K_{i} K_{i}^{*} \geq C_{2} \text {. }
$$

In both cases, we have (4.8) with $C_{1}=\min \left(C_{2}, C_{3}\right)$.

We also notice that, for each $i \geq 1$ and each $\ell=1, \ldots, n$, there exists $\tilde{a}^{\ell} \in \mathbb{R}^{n} \backslash\{0\}$ such that

$$
\kappa_{\ell}^{i}=\frac{\left(K_{i} K_{i}^{*} \tilde{a}^{\ell}, \tilde{a}^{\ell}\right)}{\left|\tilde{a}^{\ell}\right|^{2}} \leq\left\|K_{i} K_{i}^{*}\right\|_{2} \leq C_{4}\left(1+\left|\lambda_{i}\right|^{2(n-1)}\right),
$$

where we have denoted by $\|\cdot\|_{2}$ the usual Euclidean norm in $\mathcal{L}\left(\mathbb{R}^{n}\right)$. The last inequality in (4.9) is a consequence of the structure of $K_{i}$.

From (4.8) and (4.9), we see that

$$
\kappa_{1}^{i}=\frac{\operatorname{det} K_{i} K_{i}^{*}}{\Pi_{\ell \geq 2} \kappa_{\ell}^{i}} \geq \frac{C_{1}}{C_{4}^{n-1}\left(1+\left|\lambda_{i}\right|^{2(n-1)}\right)^{n-1}} \geq C_{5}\left|\lambda_{i}\right|^{-2(n-1)^{2}}
$$


and now, taking into account (4.7), the following is found:

$$
\left|K_{i}^{*} a^{i}\right|^{2}=\left(K_{i} K_{i}^{*} a^{i}, a^{i}\right) \geq \kappa_{1}^{i}\left|a^{i}\right|^{2} \geq C_{5}\left|\lambda_{i}\right|^{-2(n-1)^{2}}\left|a^{i}\right|^{2} .
$$

Finally, in view of (4.5) and (4.6), it becomes clear that, for $k \geq 2(n-1)^{2}$, one has

$$
\left\|(-\Delta)^{k} K_{*} f\right\|_{L^{2}}^{2}=\sum_{i} \lambda_{i}^{2 k}\left|K_{i}^{*} a^{i}\right|^{2} \geq C_{5} \sum_{i}\left|\lambda_{i}\right|^{2\left(k-(n-1)^{2}\right)}\left|a^{i}\right|^{2} \geq C\|f\|_{L^{2}} .
$$

Since this is true for all $f$ spanned by a finite amount of the $w^{i}$, we deduce that it must also hold for all $f \in L^{2}\left(\Omega ; \mathbb{R}^{n}\right)$ such that $(-\Delta)^{k} K_{*} f \in L^{2}\left(\Omega ; \mathbb{R}^{n}\right)$. In particular, we find (4.2).

Notice that this estimate (which is implied by (1.13)) indicates that $K_{*}$ possesses a continuous inverse in an appropriate space. The similar result we find in [5] is slightly worse, since there the restriction on $k$ is $k \geq(2 n-1)(n-1)$, see Theorem 2.1, inequality (2.12); see also ([8], Rem. 19, p. 287). A related question is whether or not an optimal lower bound of $k$ exists.

\subsection{Proof of the space-time estimate (4.3)}

For the proof of (4.3), we will use the following result, whose proof is posponed to the end of the section:

Lemma 4.1. Let us introduce the notation

$$
P\left(\partial_{t}, \Delta\right):=\operatorname{det}\left[\mathbf{I d} \partial_{t}+A^{*} \Delta+M^{*}\right] .
$$

Let $\omega_{0}$ and $\omega_{1}$ be non-empty open sets satisfying $\omega_{0} \subset \subset \omega_{1} \subset \subset \omega$, let $\alpha_{0}$ be as in (2.1) with $\omega$ replaced by $\omega_{0}$ and let us denote by $\alpha, \xi$ and $\rho$ the functions given by (2.2). There exist $s$ and $\lambda$ with the following property: for any $k \geq 0$ and $j \geq 0$, we can find an integer $m(k, j) \geq 0$, a constant $C(k, j)>0$ and an open set $\omega(k, j)$ satisfying $\omega_{0} \subset \subset \omega(k, j) \subset \subset \omega_{1}$, such that

$$
I_{12}\left(s, \lambda ;(-\Delta)^{k} \partial_{t}^{j} \phi\right) \leq C(k, j) \iint_{\omega(k, j) \times(0, T)}(t(T-t))^{-m(k, j)} \rho^{-2 s}|\phi|^{2}
$$

for any $\phi$ satisfying

$$
\partial_{t}^{i}(-\Delta)^{\ell} \phi \in L^{2}(Q), \partial_{t}^{i}(-\Delta)^{\ell} \phi=0 \text { on } \Sigma \quad \forall i, \ell \geq 0
$$

and

$$
P\left(\partial_{t}, \Delta\right) \phi=0, \quad(x, t) \in Q .
$$

Let us check that this result implies (4.3).

Let $\varphi$ be the solution to the adjoint system (1.10) (where $M$ is a constant matrix-valued function) corresponding to a final data $\varphi_{T}$, with

$$
(-\Delta)^{k} \varphi_{T} \in D(-\Delta):=H^{2}\left(\Omega ; \mathbb{R}^{n}\right) \cap H_{0}^{1}\left(\Omega ; \mathbb{R}^{n}\right) \quad \forall k \geq 0 .
$$

It is then clear that all the components of $\varphi$ satisfy (4.11).

Let us see that (for instance) the first component $\varphi_{1}$ also satisfies (4.12).

Indeed, we have:

$$
P\left(\partial_{t}, \Delta\right) \varphi_{1}=\sum_{\sigma \in \mathcal{P}_{n}}\left(p_{\sigma(1), 1} \ldots p_{\sigma(n), n}\right) \varphi_{1}
$$


where the $p_{i j}$ are the components of $\mathbf{I d} \partial_{t}+A^{*} \Delta+M^{*}$. Thus,

$$
\begin{aligned}
P\left(\partial_{t}, \Delta\right) \varphi_{1} & =\sum_{\sigma \in \mathcal{P}_{n}}\left(p_{\sigma(2), 2} \ldots p_{\sigma(n), n}\right)\left(p_{\sigma(1), 1} \varphi_{1}\right) \\
& =-\sum_{\sigma \in \mathcal{P}_{n}}\left(p_{\sigma(2), 2} \ldots p_{\sigma(n), n}\right)\left(\sum_{j=2}^{n} p_{\sigma(1), j} \varphi_{j}\right) \\
& =-\sum_{j=2}^{n}\left(\sum_{\sigma \in \mathcal{P}_{n}}\left(p_{\sigma(1), j} p_{\sigma(2), 2} \ldots p_{\sigma(n), n}\right)\right) \varphi_{j}:=-\sum_{\sigma \in \mathcal{P}_{n}} \tilde{p}_{j} \varphi_{j} .
\end{aligned}
$$

But all the operators in this last sum vanish, since each of them can be written as the determinant of a square matrix with two columns that are identical. Consequently, we certainly have

$$
P\left(\partial_{t}, \Delta\right) \varphi_{1}=0 .
$$

Obviously, this argument also holds for $\varphi_{2}, \ldots, \varphi_{n}$. Thus, we can write (4.10) for any component of $B^{*} \varphi$. This gives the following inequality for all $j, k \geq 0$ and all $\ell=1, \ldots, n^{\prime}$ :

$$
\begin{aligned}
\iint_{Q} \rho^{-2 s}\left|\left(B^{*}\left((-\Delta)^{k} \partial_{t}^{j} \varphi\right)\right)_{\ell}\right|^{2} & =\iint_{Q} \rho^{-2 s}\left|(-\Delta)^{k} \partial_{t}^{j}\left(B^{*} \varphi\right)_{\ell}\right|^{2} \\
& \leq C(k, j) \iint_{\omega \times(0, T)}\left|\left(B^{*} \phi\right)_{\ell}\right|^{2} .
\end{aligned}
$$

Let us fix $k \geq 0$. By introducing

$$
\hat{\rho}(t):=\max _{\bar{\Omega}} \rho(x, t),
$$

and recalling (4.4), we get

$$
\begin{aligned}
\iint_{Q} \hat{\rho}(t)^{-2 s}\left|(-\Delta)^{k} K_{*} \varphi\right|^{2} & \leq C \sum_{\ell=1}^{n^{\prime}} \iint_{Q} \rho^{-2 s} \mid\left(\left.B^{*}\left((-\Delta)^{k} \partial_{t}^{j} \varphi\right)_{\ell}\right|^{2}\right. \\
& \leq C \sum_{\ell=1}^{n^{\prime}} C(k, j) \iint_{\omega \times(0, T)}\left|\left(B^{*} \varphi\right)_{\ell}\right|^{2} \\
& \leq C \iint_{\omega \times(0, T)}\left|B^{*} \varphi\right|^{2}
\end{aligned}
$$

This yields (4.3).

\subsection{Conclusion and end of the proof of Theorem 1.5}

In view of $(4.2)$ and (4.3) for $k=2(n-1)^{2}$, the following holds for any solution to (1.10) associated to a final data $\varphi_{T}$ satisfying (4.13):

$$
\begin{aligned}
\iint_{Q} \hat{\rho}(t)^{-2 s}|\varphi|^{2} & =\int_{0}^{T} \hat{\rho}(t)^{-2 s}\left(\int_{\Omega}|\varphi(x, t)|^{2} \mathrm{~d} x\right) \mathrm{d} t \\
& \leq R(k) \iint_{Q} \hat{\rho}(t)^{-2 s}\left|(-\Delta)^{k}\left(K_{*} \varphi\right)\right|^{2} \\
& \leq C R(k) \iint_{\omega \times(0, T)}\left|B^{*} \varphi\right|^{2}
\end{aligned}
$$


In particular,

$$
\iint_{Q} \hat{\rho}(t)^{-2 s}|\varphi|^{2} \leq C \iint_{\omega \times(0, T)}\left|B^{*} \varphi\right|^{2} .
$$

By density, it is obvious that this inequality remains true for the solutions to (1.10) corresponding to arbitrary final data in $L^{2}\left(\Omega ; \mathbb{R}^{n}\right)$. Hence, arguing as in the final part of the proof of Theorem 1.1 in Section 2, we get (4.1), which shows that, under the assumptions of Theorem 1.5, (1.1) is null-controllable.

\subsection{Proof of Lemma 4.1}

We will adapt the arguments in [5]. The fact that $A$ is not diagonalizable introduces some nontrivial complications and the computations and estimates are more involved; but the idea is similar.

We will prove (4.10) by induction on $k$ and $j$.

\subsubsection{Step 1: Proof of (4.10) for $k=j=0$}

Let us see that, if $s$ and $\lambda$ are large enough, one has

$$
I_{12}(s, \lambda ; \phi) \leq C(0,0) \iint_{\omega(0,0) \times(0, T)}(t(T-t))^{-m(0,0)} \rho^{-2 s}|\phi|^{2}
$$

for some $m(0,0), C(0,0)$ and $\omega(0,0)$.

Again, it can be assumed that $A$ is in the Jordan canonical form. We then have for some $p \geq 1$

$$
\operatorname{Id} \partial_{t}+A^{*} \Delta+M^{*}=\left[\begin{array}{cccc}
H_{1}\left(\partial_{t}, \Delta\right) & M_{21}^{*} & \ldots & M_{p 1}^{*} \\
M_{12}^{*} & H_{2}\left(\partial_{t}, \Delta\right) & \ldots & M_{p 2}^{*} \\
\vdots & \vdots & \ddots & \vdots \\
M_{1 p}^{*} & M_{2 p}^{*} & \ldots & H_{p}\left(\partial_{t}, \Delta\right)
\end{array}\right]
$$

where we have introduced the non-scalar operators $H_{i}\left(\partial_{t}, \Delta\right):=\mathbf{I} \mathbf{d} \partial_{t}+J_{i}^{*} \Delta+M_{i i}^{*}$, the $J_{i}^{*}$ are Jordan blocks, i.e. each of them is of the form (2.7) for some $\mu_{i} \in \mathbb{C}$ and the $M_{i j}$ provide the corresponding block decomposition of $M$.

The PDE (4.12) can be written in the form

$$
\prod_{i=1}^{p} \operatorname{det} H_{i}\left(\partial_{t}, \Delta\right) \phi=F(\phi) .
$$

In the terms in $F(\phi)$ we find the composition of at most $p-2$ operators of the kind $\operatorname{det} H_{j}\left(\partial_{t}, \Delta\right)$ applied to $\phi$.

Let us introduce the functions $\psi_{i}$, with

$$
\psi_{1}=\phi, \psi_{2}=\operatorname{det} H_{1}\left(\partial_{t}, \Delta\right) \psi_{1}, \ldots, \psi_{p}=\operatorname{det} H_{p-1}\left(\partial_{t}, \Delta\right) \psi_{p-1} .
$$

Then we can rewrite (4.15) as the following system for the $\psi_{i}$ :

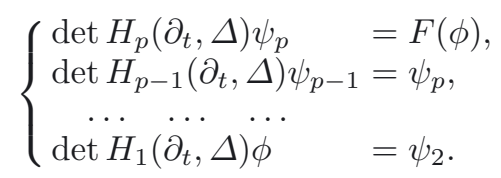

Recall that, by hypothesis, we also have

$$
\phi=\psi_{2}=\ldots=\psi_{p}=0 \text { on } \Sigma .
$$

We will now provide global estimates of $\psi_{p}$ and its derivatives in terms of local estimates of $\psi_{p}$ and (lower order) estimates of $F(\phi)$; then, global estimates of $\psi_{p-1}$ and its derivatives in terms of local estimates of $\psi_{p-1}$ 
and lower order estimates of $\psi_{p}$; etc. And, finally, global estimates of $\phi$ and its derivatives in terms of local estimates of $\phi$ and (lower order) estimates of $\psi_{2}$. An appropriate combination of these estimates will lead to an inequality where we find, in the left hand side, global weighted integrals of $\phi, \psi_{2}, \ldots$ and, in the right, only local integrals and a global weighted integral of $|F(\phi)|^{2}$.

Thus, let us consider the first PDE in (4.16). For instance, assume that $J_{p}$ is a Jordan block of dimension $r$ associated to the complex eigenvalue $\alpha$ with $\Re \alpha>0$ and let us denote by $\eta_{1}, \ldots, \eta_{r}$ the diagonal components of $M_{p p}$; by assumption, $r \leq 4$. Then, this PDE can be rewritten in the form

$$
\prod_{i=1}^{r}\left(\partial_{t}+\alpha \Delta+\eta_{i}\right) \psi_{p}=F(\phi)-G\left(\psi_{p}\right),
$$

where $G\left(\psi_{p}\right)$ is a linear combination of partial derivatives of $\psi_{p}$.

Let us introduce the new variables

$$
\zeta_{1}=\psi_{p}, \zeta_{2}=\left(\partial_{t}+\alpha \Delta+\eta_{r}\right) \zeta_{1}, \ldots, \zeta_{r}=\left(\partial_{t}+\alpha \Delta+\eta_{2}\right) \zeta_{r-1}
$$

Now, we can rewrite (4.18) as a first-order system for the $\zeta_{i}$ :

$$
\left\{\begin{array}{c}
\left(\partial_{t}+\alpha \Delta+\eta_{1}\right) \zeta_{r}=F(\phi)-G\left(\psi_{p}\right), \\
\left(\partial_{t}+\alpha \Delta+\eta_{2}\right) \zeta_{r-1}=\zeta_{r}, \\
\cdots \cdots \cdots \\
\left(\partial_{t}+\alpha \Delta+\eta_{r}\right) \zeta_{1}=\zeta_{2} .
\end{array}\right.
$$

Again, we have information on the $\zeta_{i}$ on the lateral boundary:

$$
\zeta_{1}=\ldots=\zeta_{r}=0 \text { on } \Sigma .
$$

In the "worst" possible case, we have $r=4$ and (4.19) and (4.20) respectively read

$$
\left\{\begin{array}{l}
\left(\partial_{t}+\alpha \Delta+\eta_{1}\right) \zeta_{4}=F(\phi)-G\left(\psi_{p}\right), \\
\left(\partial_{t}+\alpha \Delta+\eta_{2}\right) \zeta_{3}=\zeta_{4}, \\
\left(\partial_{t}+\alpha \Delta+\eta_{3}\right) \zeta_{2}=\zeta_{3}, \\
\left(\partial_{t}+\alpha \Delta+\eta_{4}\right) \zeta_{1}=\zeta_{2},
\end{array}\right.
$$

and

$$
\zeta_{1}=\zeta_{2}=\zeta_{3}=\zeta_{4}=0 \text { on } \Sigma \text {. }
$$

Notice that $\left|G\left(\psi_{p}\right)\right|^{2}$ is bounded by a sum of squares of derivatives of $\psi_{p}$. More precisely, we have $\left|G\left(\psi_{p}\right)\right|^{2} \leq$ $C I_{G}\left(\psi_{p}\right)$, with

$$
\begin{aligned}
I_{G}\left(\psi_{p}\right):= & \sum_{a=0}^{3}\left|(-\Delta)^{a} \psi_{p}\right|^{2}+\sum_{j=1}^{4} \sum_{b=0}^{2}\left|(-\Delta)^{b}\left(\partial_{t}+\alpha \Delta+\eta_{j}\right) \psi_{p}\right|^{2} \\
& +\sum_{j, k=1}^{4} \sum_{c=0}^{1}\left|(-\Delta)^{c}\left(\partial_{t}+\alpha \Delta+\eta_{j}\right)\left(\partial_{t}+\alpha \Delta+\eta_{k}\right) \psi_{p}\right|^{2} .
\end{aligned}
$$

In view of the Carleman estimates (2.4) (established in Lem. 2.2) applied to the functions $\zeta_{i}$, we have:

$$
\begin{aligned}
I_{3}\left(s, \lambda ; \zeta_{4}\right) & \leq C \iint_{Q} \rho^{-2 s}\left(|F(\phi)|^{2}+I_{G}\left(\psi_{p}\right)\right)+C I_{3, \omega_{0}}\left(s, \lambda ; \zeta_{4}\right), \\
\lambda^{4} I_{6}\left(s, \lambda ; \zeta_{3}\right) & \leq C \iint_{Q} \rho^{-2 s}(s \xi)^{3} \lambda^{4}\left|\zeta_{4}\right|^{2}+C \lambda^{4} I_{6, \omega_{0}}\left(s, \lambda ; \zeta_{3}\right), \\
\lambda^{8} I_{9}\left(s, \lambda ; \zeta_{2}\right) & \leq C \iint_{Q} \rho^{-2 s}(s \xi)^{6} \lambda^{8}\left|\zeta_{3}\right|^{2}+C \lambda^{8} I_{9, \omega_{0}}\left(s, \lambda ; \zeta_{2}\right), \\
\lambda^{12} I_{12}\left(s, \lambda ; \zeta_{1}\right) & \leq C \iint_{Q} \rho^{-2 s}(s \xi)^{9} \lambda^{12}\left|\zeta_{2}\right|^{2}+C \lambda^{12} I_{12, \omega_{0}}\left(s, \lambda ; \zeta_{1}\right),
\end{aligned}
$$

for any $\lambda \geq \lambda_{0}$ and $s \geq s_{0}=\sigma_{0}\left(T+T^{2}\right)$. 
Consequently, an appropriate linear combination of the terms in the left hand sides absorbes the global weighted integrals of $\left|\zeta_{4}\right|^{2},\left|\zeta_{3}\right|^{2}$ and $\left|\zeta_{2}\right|^{2}$. More precisely, for $s \geq s_{0}$ and $\lambda \geq \lambda_{0}$, we get:

$$
\begin{aligned}
& I_{3}\left(s, \lambda ; \zeta_{4}\right)+\lambda^{4} I_{6}\left(s, \lambda ; \zeta_{3}\right)+\lambda^{8} I_{9}\left(s, \lambda ; \zeta_{2}\right)+\lambda^{12} I_{12}\left(s, \lambda ; \zeta_{1}\right) \\
& \leq C\left(I_{3, \omega_{0}}\left(s, \lambda ; \zeta_{4}\right)+\lambda^{4} I_{6, \omega_{0}}\left(s, \lambda ; \zeta_{3}\right)+\lambda^{8} I_{9, \omega_{0}}\left(s, \lambda ; \zeta_{2}\right)+\lambda^{12} I_{12, \omega_{0}}\left(s, \lambda ; \zeta_{1}\right)\right) \\
& +C \iint_{Q} \rho^{-2 s}|F(\phi)|^{2}+C \iint_{Q} \rho^{-2 s} I_{G}\left(\psi_{p}\right) .
\end{aligned}
$$

The next task will be to add some extra terms on the left hand side of the previous inequality. To this end, we reason as follow. We apply $-\Delta$ to the second PDE in (4.21) and we use Lemma 2.1 for the resulting equation with $m=2$. Notice that this is possible, since $-\Delta \zeta_{3}=0$ on $\Sigma$. We find:

$$
\begin{aligned}
I_{2}\left(s, \lambda ; \Delta \zeta_{3}\right) & \left.\leq C \iint_{\omega_{0} \times(0, T)} \rho^{-2 s}(s \xi)^{2} \lambda^{4}\left|\Delta \zeta_{3}\right|^{2}\right)+C \iint_{Q} \rho^{-2 s}(s \xi)^{-1}\left|\Delta \zeta_{4}\right|^{2} \\
& \leq C\left(\lambda^{4} I_{6}\left(s, \lambda ; \zeta_{3}\right)+I_{3}\left(s, \lambda ; \zeta_{4}\right)\right) .
\end{aligned}
$$

Observe that the previous argument can be applied, this time, to the third and fourth PDE in (4.21). Applying $-\Delta$ and using Lemma 2.1 for the corresponding equations with $m=5$ and $m=8$, we deduce:

$$
\begin{aligned}
\lambda^{4} I_{5}\left(s, \lambda ; \Delta \zeta_{2}\right) & \left.\leq C \iint_{\omega_{0} \times(0, T)} \rho^{-2 s}(s \xi)^{5} \lambda^{8}\left|\Delta \zeta_{2}\right|^{2}\right)+C \iint_{Q} \rho^{-2 s}(s \xi)^{2} \lambda^{4}\left|\Delta \zeta_{3}\right|^{2} \\
& \leq C\left(\lambda^{8} I_{9}\left(s, \lambda ; \zeta_{2}\right)+\lambda^{4} I_{6}\left(s, \lambda ; \zeta_{3}\right)\right)
\end{aligned}
$$

and

$$
\begin{aligned}
\lambda^{8} I_{8}\left(s, \lambda ; \Delta \zeta_{1}\right) & \left.\leq C \iint_{\omega_{0} \times(0, T)} \rho^{-2 s}(s \xi)^{8} \lambda^{12}\left|\Delta \zeta_{1}\right|^{2}\right)+C \iint_{Q} \rho^{-2 s}(s \xi)^{5} \lambda^{8}\left|\Delta \zeta_{2}\right|^{2} \\
& \leq C\left(\lambda^{12} I_{12}\left(s, \lambda ; \zeta_{1}\right)+\lambda^{8} I_{8}\left(s, \lambda ; \zeta_{2}\right)\right) .
\end{aligned}
$$

Then, we can add all these new terms to the left hand side of (4.23) and, for a new positive constant $C$, obtain

$$
\begin{aligned}
& I_{3}\left(s, \lambda ; \zeta_{4}\right)+\lambda^{4} I_{6}\left(s, \lambda ; \zeta_{3}\right)+\lambda^{8} I_{9}\left(s, \lambda ; \zeta_{2}\right)+\lambda^{12} I_{12}\left(s, \lambda ; \zeta_{1}\right) \\
& \quad+I_{2}\left(s, \lambda ; \Delta \zeta_{3}\right)+\lambda^{4} I_{5}\left(s, \lambda ; \Delta \zeta_{2}\right)+\lambda^{8} I_{8}\left(s, \lambda ; \Delta \zeta_{1}\right) \\
& \leq \\
& \quad C\left(I_{3, \omega_{0}}\left(s, \lambda ; \zeta_{4}\right)+\lambda^{4} I_{6, \omega_{0}}\left(s, \lambda ; \zeta_{3}\right)+\lambda^{8} I_{9, \omega_{0}}\left(s, \lambda ; \zeta_{2}\right)+\lambda^{12} I_{12, \omega_{0}}\left(s, \lambda ; \zeta_{1}\right)\right) \\
& \quad+C \iint_{Q} \rho^{-2 s}|F(\phi)|^{2}+C \iint_{Q} \rho^{-2 s} I_{G}\left(\psi_{p}\right) .
\end{aligned}
$$

We can continue the previous process and add better global terms on the left hand side of (4.24). Thus, if we apply $(-\Delta)^{2}$ to the third PDE in (4.21) and we use again Lemma 2.1 for the corresponding equations with $m=1$, we get

$$
\begin{aligned}
I_{1}\left(s, \lambda ;(-\Delta)^{2} \zeta_{2}\right) & \left.\leq C \iint_{\omega_{0} \times(0, T)} \rho^{-2 s} s \xi \lambda^{4}\left|(-\Delta)^{2} \zeta_{2}\right|^{2}\right)+C \iint_{Q} \rho^{-2 s}(s \xi)^{-2}\left|\Delta \zeta_{3}\right|^{2} \\
& \leq C\left(\lambda^{4} I_{5}\left(s, \lambda ; \Delta \zeta_{2}\right)+I_{2}\left(s, \lambda ; \Delta \zeta_{3}\right)\right)
\end{aligned}
$$

The previous argument, this time applied to the last PDE in (4.21), also gives

$$
\lambda^{4} I_{4}\left(s, \lambda ;(-\Delta)^{2} \zeta_{1}\right) \leq C\left(\lambda^{8} I_{8}\left(s, \lambda ; \Delta \zeta_{1}\right)+\lambda^{8} I_{5}\left(s, \lambda ; \Delta \zeta_{2}\right)\right) .
$$


Putting the previous inequalities in (4.24), we obtain

$$
\begin{aligned}
& I_{3}\left(s, \lambda ; \zeta_{4}\right)+\lambda^{4} I_{6}\left(s, \lambda ; \zeta_{3}\right)+\lambda^{8} I_{9}\left(s, \lambda ; \zeta_{2}\right)+\lambda^{12} I_{12}\left(s, \lambda ; \zeta_{1}\right) \\
& +I_{2}\left(s, \lambda ; \Delta \zeta_{3}\right)+\lambda^{4} I_{5}\left(s, \lambda ; \Delta \zeta_{2}\right)+\lambda^{8} I_{8}\left(s, \lambda ; \Delta \zeta_{1}\right) \\
& +I_{1}\left(s, \lambda ;(-\Delta)^{2} \zeta_{2}\right)+\lambda^{4} I_{4}\left(s, \lambda ;(-\Delta)^{2} \zeta_{1}\right) \\
& \leq C\left(I_{3, \omega_{0}}\left(s, \lambda ; \zeta_{4}\right)+\lambda^{4} I_{6, \omega_{0}}\left(s, \lambda ; \zeta_{3}\right)+\lambda^{8} I_{9, \omega_{0}}\left(s, \lambda ; \zeta_{2}\right)+\lambda^{12} I_{12, \omega_{0}}\left(s, \lambda ; \zeta_{1}\right)\right) \\
& +C \iint_{Q} \rho^{-2 s}|F(\phi)|^{2}+C \iint_{Q} \rho^{-2 s} I_{G}\left(\psi_{p}\right) .
\end{aligned}
$$

Finally, let us take $(-\Delta)^{3}$ to the last PDE of (4.21). Again, from the regularity assumptions on $\phi$ we have $(-\Delta)^{3} \zeta_{1}=0$ on $\Sigma$. So, we can apply Lemma 2.1 with $m=0$ to the resulting PDE and deduce

$$
I_{0}\left(s, \lambda ;(-\Delta)^{3} \zeta_{1}\right) \leq C\left(\lambda^{4} I_{4}\left(s, \lambda ;(-\Delta)^{2} \zeta_{1}\right)+I_{1}\left(s, \lambda ;(-\Delta)^{2} \zeta_{2}\right)\right) .
$$

This inequality together with (4.25) provides

$$
\begin{aligned}
& I_{3}\left(s, \lambda ; \zeta_{4}\right)+\lambda^{4} I_{6}\left(s, \lambda ; \zeta_{3}\right)+\lambda^{8} I_{9}\left(s, \lambda ; \zeta_{2}\right)+\lambda^{12} I_{12}\left(s, \lambda ; \zeta_{1}\right) \\
& \quad+I_{2}\left(s, \lambda ; \Delta \zeta_{3}\right)+\lambda^{4} I_{5}\left(s, \lambda ; \Delta \zeta_{2}\right)+\lambda^{8} I_{8}\left(s, \lambda ; \Delta \zeta_{1}\right) \\
& \quad+I_{1}\left(s, \lambda ;(-\Delta)^{2} \zeta_{2}\right)+\lambda^{4} I_{4}\left(s, \lambda ;(-\Delta)^{2} \zeta_{1}\right) \\
& \quad+I_{0}\left(s, \lambda ;(-\Delta)^{3} \zeta_{1}\right) \\
& \leq \\
& \quad C\left(I_{3, \omega_{0}}\left(s, \lambda ; \zeta_{4}\right)+\lambda^{4} I_{6, \omega_{0}}\left(s, \lambda ; \zeta_{3}\right)+\lambda^{8} I_{9, \omega_{0}}\left(s, \lambda ; \zeta_{2}\right)+\lambda^{12} I_{12, \omega_{0}}\left(s, \lambda ; \zeta_{1}\right)\right) \\
& \quad+C \iint_{Q} \rho^{-2 s}|F(\phi)|^{2}+C \iint_{Q} \rho^{-2 s} I_{G}\left(\psi_{p}\right),
\end{aligned}
$$

for a new positive constant $C$. Let us denote by $J_{\text {tot }}(s, \lambda ; \zeta)$ the sum of the terms on the left hand side of the previous inequality (with $\left.\zeta=\left(\zeta_{1}, \zeta_{2}, \zeta_{3}, \zeta_{4}\right)\right)$. Then, the previous inequality can be written as

$$
\begin{aligned}
J_{\text {tot }}(s, \lambda ; \zeta) \leq & C\left(I_{3, \omega_{0}}\left(s, \lambda ; \zeta_{4}\right)+\lambda^{4} I_{6, \omega_{0}}\left(s, \lambda ; \zeta_{3}\right)+\lambda^{8} I_{9, \omega_{0}}\left(s, \lambda ; \zeta_{2}\right)\right. \\
& \left.+\lambda^{12} I_{12, \omega_{0}}\left(s, \lambda ; \zeta_{1}\right)\right)+C\left(\iint_{Q} \rho^{-2 s}|F(\phi)|^{2}+\iint_{Q} \rho^{-2 s} I_{G}\left(\psi_{p}\right)\right),
\end{aligned}
$$

with $s \geq s_{1}=\sigma_{1}\left(T+T^{2}\right)$ and $\lambda \geq \lambda_{1}$.

Let $\omega(0,0)$ be an open set satisfying $\omega_{0} \subset \subset \omega(0,0) \subset \subset \omega_{1}$. From now on, we fix $s \geq s_{1}$ and $\lambda \geq \lambda_{1}$ and we try to replace the local terms in (4.26) corresponding to $\zeta_{2}, \zeta_{3}$ and $\zeta_{4}$ by a term of the form $\left(\psi_{p}=\zeta_{1}\right)$

$$
C \lambda^{\ell_{1}} \iint_{\omega(0,0) \times(0, T)} \rho^{-2 s}(s \xi)^{\ell_{2}}\left|\psi_{p}\right|^{2},
$$

where $\ell_{1}$ and $\ell_{2}$ are nonnegative integers. We need some lengthy computations, but using the cascade structure of the system (4.21), the process is clear. For instance, let us see what can be done with the local term corresponding to $\zeta_{4}$.

First, we introduce an open subset $\widetilde{\omega}_{0}$, with $\omega_{0} \subset \subset \widetilde{\omega}_{0} \subset \subset \omega(0,0)$, and a cut-off function $\chi=\chi(x)$ with $\chi \in C_{0}^{\infty}\left(\widetilde{\omega}_{0}\right), \chi \geq 0$ and $\chi \equiv 1$ in $\omega_{0}$ and we write $\zeta_{4}=\left(\partial_{t}+\alpha \Delta+\eta_{2}\right) \zeta_{3}$ (see $\left.(4.21)\right)$

$$
\begin{aligned}
I_{3, \omega_{0}}\left(s, \lambda ; \zeta_{4}\right) & \leq \lambda^{4} \iint_{\widetilde{\omega}_{0} \times(0, T)} \rho^{-2 s}(s \xi)^{3} \chi\left|\zeta_{4}\right|^{2} \\
& =\lambda^{4} \iint_{\widetilde{\omega}_{0} \times(0, T)} \rho^{-2 s}(s \xi)^{3} \chi\left(\partial_{t}+\alpha \Delta+\eta_{2}\right) \zeta_{3} \bar{\zeta}_{4}=I_{1}+I_{2}+I_{3} .
\end{aligned}
$$


Let us take $\mu$ in (2.2) in such a way we have

$$
\mathrm{e}^{(\lambda+\mu)\left\|\alpha_{0}\right\|_{L} \infty}-\mathrm{e}^{\lambda \alpha_{0}(x)} \geq \mathrm{e}^{\lambda \alpha_{0}(x)} \geq 1, \quad \forall x \in \Omega .
$$

Then, we also have

$$
\left\{\begin{array}{l}
\left|\nabla\left(\rho^{-2 s}(s \xi)^{\ell}\right)\right| \leq C \lambda \rho^{-2 s}(s \xi)^{\ell+1},\left|\Delta\left(\rho^{-2 s}(s \xi)^{\ell}\right)\right| \leq C \lambda^{2} \rho^{-2 s}(s \xi)^{\ell+2}, \\
\left|\partial_{t}\left(\rho^{-2 s}(s \xi)^{\ell}\right)\right| \leq C \rho^{-2 s}(s \xi)^{\ell+2}
\end{array}\right.
$$

for any $(x, t) \in Q$ and $s \geq s_{1}$ and $\lambda \geq \lambda_{1}$.

Using the previous inequalities, we deduce

$$
\begin{aligned}
I_{1} & =-\lambda^{4} \iint_{\widetilde{\omega}_{0} \times(0, T)} \partial_{t}\left(\rho^{-2 s}(s \xi)^{3}\right) \chi \zeta_{3} \bar{\zeta}_{4}-\lambda^{4} \iint_{\widetilde{\omega}_{0} \times(0, T)} \rho^{-2 s}(s \xi)^{3} \chi \zeta_{3} \partial_{t} \bar{\zeta}_{4} \\
& \leq C \lambda^{4} \iint_{\widetilde{\omega}_{0} \times(0, T)} \rho^{-2 s}(s \xi)^{5} \chi \zeta_{3} \bar{\zeta}_{4}+\lambda^{4} \iint_{\widetilde{\omega}_{0} \times(0, T)} \rho^{-2 s}(s \xi)^{3} \chi \zeta_{3} \partial_{t} \bar{\zeta}_{4} \\
& \leq \varepsilon I_{3}\left(s, \lambda ; \zeta_{4}\right)+\frac{C}{\varepsilon} \lambda^{8} \iint_{\widetilde{\omega}_{0} \times(0, T)} \rho^{-2 s}(s \xi)^{7}\left|\zeta_{3}\right|^{2},
\end{aligned}
$$

with $\varepsilon>0$.

We can also bound

$$
\begin{aligned}
I_{2}= & \alpha \lambda^{4} \iint_{\widetilde{\omega}_{0} \times(0, T)} \Delta\left(\rho^{-2 s}(s \xi)^{3} \chi \bar{\zeta}_{4}\right) \zeta_{3} \\
= & \alpha \lambda^{4} \iint_{\widetilde{\omega}_{0} \times(0, T)}\left[\Delta\left(\rho^{-2 s}(s \xi)^{3}\right) \bar{\zeta}_{4}+2 \nabla\left(\rho^{-2 s}(s \xi)^{3}\right) \cdot \nabla \bar{\zeta}_{4}\right] \chi \zeta_{3} \\
& +\alpha \lambda^{4} \iint_{\widetilde{\omega}_{0} \times(0, T)} \rho^{-2 s}(s \xi)^{3} \Delta \bar{\zeta}_{4} \chi \zeta_{3}+\ldots
\end{aligned}
$$

where the dots contain terms of lower order. Now, we apply the Cauchy-Schwarz inequality and inequality (4.27). So,

$$
I_{2} \leq \varepsilon I_{3}\left(s, \lambda ; \zeta_{4}\right)+\frac{C}{\varepsilon} \lambda^{8} \iint_{\widetilde{\omega}_{0} \times(0, T)} \rho^{-2 s}(s \xi)^{7}\left|\zeta_{3}\right|^{2} .
$$

Finally,

$$
I_{3} \leq \varepsilon I_{3}\left(s, \lambda ; \zeta_{4}\right)+\frac{C}{\varepsilon} \lambda^{4} \iint_{\widetilde{\omega}_{0} \times(0, T)} \rho^{-2 s}(s \xi)^{3}\left|\zeta_{3}\right|^{2} .
$$

Putting the previous inequalities together, we get

$$
I_{3, \omega_{0}}\left(s, \lambda ; \zeta_{4}\right) \leq \varepsilon I_{3}\left(s, \lambda ; \zeta_{4}\right)+\frac{C}{\varepsilon} \lambda^{4} \iint_{\widetilde{\omega}_{0} \times(0, T)} \rho^{-2 s}(s \xi)^{7}\left|\zeta_{3}\right|^{2} .
$$

The previous inequality is valid for any $\varepsilon>0, s \geq s_{1}$ and $\lambda \geq \lambda_{1}$.

Coming back to (4.26), if we take $\varepsilon$ small enough, we obtain

$$
\begin{aligned}
J_{\text {tot }}(s, \lambda ; \zeta) \leq & C\left(\lambda^{8} I_{7, \tilde{\omega}_{0}}\left(s, \lambda ; \zeta_{3}\right)+\lambda^{8} I_{9, \omega_{0}}\left(s, \lambda ; \zeta_{2}\right)+\lambda^{12} I_{12, \omega_{0}}\left(s, \lambda ; \zeta_{1}\right)\right) \\
& +C\left(\iint_{Q} \rho^{-2 s}|F(\phi)|^{2}+\iint_{Q} \rho^{-2 s} I_{G}\left(\psi_{p}\right)\right)
\end{aligned}
$$

for a new positive constant $C$. 
Observe that the previous reasoning can be applied twice in order to eliminate the local terms corresponding to $\zeta_{3}$ and $\zeta_{2}$. The resulting inequality is

$$
J_{\text {tot }}(s, \lambda ; \zeta) \leq C \lambda^{\ell_{1}} \iint_{\widetilde{\omega}_{1} \times(0, T)} \rho^{-2 s}(s \xi)^{\ell_{2}}\left|\psi_{p}\right|^{2}+C\left(\iint_{Q} \rho^{-2 s}|F(\phi)|^{2}+\iint_{Q} \rho^{-2 s} I_{G}\left(\psi_{p}\right)\right),
$$

with $s \geq s_{1}=\sigma_{1}\left(T+T^{2}\right)$ and $\lambda \geq \lambda_{1}$. In inequality (4.28) $\widetilde{\omega}_{1}$ is a new open subset satisfying $\widetilde{\omega}_{0} \subset \subset \widetilde{\omega}_{1} \subset \subset \omega(0,0)$ and $\ell_{1}$ and $\ell_{2}$ are nonnegative intergers.

Now, taking into account that the operators $\partial_{t}+\alpha \Delta+\eta_{i}$ commute, we see that (4.18) (for $r=4$ ) can be rewritten equivalently in the form

$$
\prod_{i=1}^{4}\left(\partial_{t}+\alpha \Delta+\eta_{\sigma(i)}\right) \psi_{p}=F(\phi)-G\left(\psi_{p}\right)
$$

where $\sigma$ is any permutation in $\mathcal{P}_{4}$. Hence, we can introduce the new variables

$$
\zeta_{1}^{\sigma}=\psi_{p}, \zeta_{2}^{\sigma}=\left(\partial_{t}+\alpha \Delta+\eta_{\sigma(4)}\right) \zeta_{1}^{\sigma}, \ldots, \zeta_{4}^{\sigma}=\left(\partial_{t}+\alpha \Delta+\eta_{\sigma(2)}\right) \zeta_{3}^{\sigma}
$$

and we can also write (4.18) as a similar first-order system for the $\zeta_{i}^{\sigma}$ :

$$
\left\{\begin{array}{l}
\left(\partial_{t}+\alpha \Delta+\eta_{\sigma(1)}\right) \zeta_{4}^{\sigma}=F(\phi)-G\left(\psi_{p}\right) \\
\left(\partial_{t}+\alpha \Delta+\eta_{\sigma(2)}\right) \zeta_{3}^{\sigma}=\zeta_{4}^{\sigma} \\
\left(\partial_{t}+\alpha \Delta+\eta_{\sigma(3)}\right) \zeta_{2}^{\sigma}=\zeta_{3}^{\sigma} \\
\left(\partial_{t}+\alpha \Delta+\eta_{\sigma(4)}\right) \zeta_{1}^{\sigma}=\zeta_{2}^{\sigma}
\end{array}\right.
$$

Again, we have:

$$
\zeta_{1}^{\sigma}=\zeta_{2}^{\sigma}=\zeta_{3}^{\sigma}=\zeta_{4}^{\sigma}=0 \text { on } \Sigma .
$$

Arguing as before, we obtain an estimate like (4.28) where, now, we have in the left global weighted integrals of $\zeta_{1}^{\sigma}=\psi_{p}, \zeta_{2}^{\sigma}, \zeta_{3}^{\sigma}$ and $\zeta_{4}^{\sigma}$ and, in the right, terms concerning $F(\phi)$ and $I_{G}\left(\psi_{p}\right)$ :

$$
\begin{aligned}
J_{\text {tot }}\left(s, \lambda ; \zeta^{\sigma}\right) \leq & C \lambda^{\ell_{1}} \iint_{\widetilde{\omega}_{1} \times(0, T)} \rho^{-2 s}(s \xi)^{\ell_{2}}\left|\psi_{p}\right|^{2} \\
& +C\left(\iint_{Q} \rho^{-2 s}|F(\phi)|^{2}+\iint_{Q} \rho^{-2 s} I_{G}\left(\psi_{p}\right)\right) .
\end{aligned}
$$

In this inequality we have used the notation $\zeta^{\sigma}=\left(\zeta_{1}^{\sigma}, \zeta_{2}^{\sigma}, \zeta_{3}^{\sigma}, \zeta_{1}^{\sigma}\right)$.

Let us denote by $I_{\text {tot }}\left(s, \lambda ; \psi_{p}\right)$ the sum of all these left hand sides, obtained for all $\sigma \in \mathcal{P}_{4}$. Then

$$
\begin{aligned}
I_{\text {tot }}\left(s, \lambda ; \psi_{p}\right) \leq & C \lambda^{\ell_{1}} \iint_{\widetilde{\omega}_{1} \times(0, T)} \rho^{-2 s}(s \xi)^{\ell_{2}}\left|\psi_{p}\right|^{2} \\
& +C\left(\iint_{Q} \rho^{-2 s}|F(\phi)|^{2}+\iint_{Q} \rho^{-2 s} I_{G}\left(\psi_{p}\right)\right) .
\end{aligned}
$$

Observe that all the terms in $I_{G}\left(\psi_{p}\right)$ except $\left|(-\Delta)^{3} \psi_{p}\right|^{2}$ are also in the left multiplied by weights of the form $(s \xi)^{a} \rho^{-2 s}$ with $a>0$. Consequently, for sufficiently large $s$, these terms are absorbed and we find:

$$
\begin{aligned}
I_{\text {tot }}\left(s, \lambda ; \psi_{p}\right) \leq & C \lambda^{\ell_{1}} \iint_{\widetilde{\omega}_{1} \times(0, T)} \rho^{-2 s}(s \xi)^{\ell_{2}}\left|\psi_{p}\right|^{2} \\
& +C \iint_{Q} \rho^{-2 s}|F(\phi)|^{2}+C \iint_{Q} \rho^{-2 s}\left|(-\Delta)^{3} \psi_{p}\right|^{2} .
\end{aligned}
$$


Finally the global term corresponding to $\rho^{-2 s}\left|(-\Delta)^{3} \psi_{p}\right|^{2}$ can be absorbed by the term $I_{0}\left(s, \lambda ;(-\Delta)^{3} \psi_{p}\right)$ (appearing in the expression of $\left.I_{\text {tot }}\left(s, \lambda ; \psi_{p}\right)\right)$ taking $\lambda$ large enough. Hence,

$$
\begin{aligned}
I_{\text {tot }}\left(s, \lambda ; \psi_{p}\right) \leq & C \lambda^{\ell_{1}} \iint_{\widetilde{\omega}_{1} \times(0, T)} \rho^{-2 s}(s \xi)^{\ell_{2}}\left|\psi_{p}\right|^{2} \\
& +C \iint_{Q} \rho^{-2 s}|F(\phi)|^{2}, \quad \forall s \geq s_{2}=\sigma_{2}\left(T+T^{2}\right), \quad \lambda \geq \lambda_{2},
\end{aligned}
$$

for some $n(0,0), m(0,0) \geq 1$.

From now on, we fix $\lambda=\lambda_{2}$. Let us now consider the second PDE in (4.16). Arguing in the same way (and assuming again that we are in the worst possible situation, associated to a block of dimension 4), we deduce the following estimate for $\psi_{p-1}$ :

$$
I_{12}\left(s, \lambda ; \psi_{p-1}\right) \leq C\left(\iint_{Q} \rho^{-2 s}\left|\psi_{p}\right|^{2}+\iint_{\widetilde{\omega}_{1} \times(0, T)}(s \xi)^{\ell_{2}} \rho^{-2 s}\left|\psi_{p-1}\right|^{2}\right) .
$$

The corresponding similar estimate also holds for $\psi_{p-2}$, etc. Thus, after addition and taking into account that $\psi_{1}=\phi$ and the global integrals of $\psi_{p}, \ldots, \psi_{2}$ in the right hand side are smaller than the terms in the left, we get an estimate for all the $\psi_{i}$ :

$$
\sum_{i=1}^{p} I_{12}\left(s, \lambda ; \psi_{i}\right) \leq C\left(\iint_{Q} \rho^{-2 s}|F(\phi)|^{2}+\iint_{\widetilde{\omega}_{1} \times(0, T)}(s \xi)^{\ell_{2}} \rho^{-2 s}|\phi|^{2}+\sum_{i=2}^{p} \iint_{\widetilde{\omega}_{1} \times(0, T)}(s \xi)^{\ell_{2}} \rho^{-2 s}\left|\psi_{i}\right|^{2}\right) .
$$

Again, using the cascade structure of system (4.16), all the local integrals in the right can be absorbed by the left hand side, with the exception of the local weighted integral of $|\phi|^{2}$. All we have to do is to enlarge the open set $\widetilde{\omega}_{1}$ and argue like in the passage from (4.26) to (4.28). Therefore, the following is obtained:

$$
\sum_{i=1}^{p} I_{12}\left(s, \lambda ; \psi_{i}\right) \leq C\left(\iint_{Q} \rho^{-2 s}|F(\phi)|^{2}+\iint_{\omega(0,0) \times(0, T)}(s \xi)^{m(0,0)} \rho^{-2 s} \chi|\phi|^{2}\right) .
$$

However, we see that, taking into account that the operators det $H_{i}\left(\partial_{t}, \Delta\right)$ commute, (4.16) can also be written in the form

$$
\prod_{i=1}^{p} \operatorname{det} H_{\sigma(i)}\left(\partial_{t}, \Delta\right) \phi=F(\phi)
$$

where $\sigma$ is any permutation in $\mathcal{P}_{n}$. This means that another equivalent formulation of $(4.12)$ is

$$
\left\{\begin{array}{cc}
\operatorname{det} H_{\sigma(p)}\left(\partial_{t}, \Delta\right) \psi_{p}^{\sigma} & =F(\phi), \\
\operatorname{det} H_{\sigma(p-1)}\left(\partial_{t}, \Delta\right) \psi_{p-1}^{\sigma} & =\psi_{p}^{\sigma}, \\
\ldots \quad \cdots & \cdots \\
\operatorname{det} H_{\sigma(1)}\left(\partial_{t}, \Delta\right) \phi & =\psi_{2}^{\sigma},
\end{array}\right.
$$

and we can also get an estimate of the same form where, now, we have in the left global weighted integrals of $\phi, \psi_{2}^{\sigma}, \ldots, \psi_{p}^{\sigma}$. Recall that $F(\phi)$ is a sum of terms where, at most, $p-2$ operators of the kind $\operatorname{det} H_{j}\left(\partial_{t}, \Delta\right)$ are applied to $\phi$. Since $\sigma$ is arbitrary in $\mathcal{P}_{n}$, using all these possible estimates together and arguing as above, it becomes also clear that the terms containing $|F(\phi)|^{2}$ can be controlled by the terms in the left.

This proves (4.14). 


\subsubsection{Step 2: Induction on $k$ and $j$}

Now, let us assume that (4.10) is true for any $k^{\prime}=0,1, \ldots, k$, any $j^{\prime}=0,1, \ldots, j$ and any solution to (4.12) satisfying the assumptions of the lemma and let us prove (4.10) (for instance) with $k$ replaced by $k+1$; the proof with the same $k$ and $j$ replaced by $j+1$ is essentially the same.

Since $\hat{\phi}:=(-\Delta) \phi$ also satisfies (4.11) and (4.12), we have by hypothesis

$$
I_{12}\left(s, \lambda ;(-\Delta)^{k} \partial_{t}^{j} \hat{\phi}\right) \leq C(k, j) \iint_{\omega(k, j) \times(0, T)}(t(T-t))^{-m(k, j)} \rho^{-2 s}|\hat{\phi}|^{2},
$$

that is,

$$
I_{12}\left(s, \lambda ;(-\Delta)^{k+1} \partial_{t}^{j} \phi\right) \leq C(k, j) \iint_{\omega(k, j) \times(0, T)}(t(T-t))^{-m(k, j)} \rho^{-2 s}|\Delta \phi|^{2} .
$$

Let us set $\tilde{m}=m(k, j)$ and $\tilde{\omega}=\omega(k, j)$, let $\omega^{*}$ be an open set satisfying $\tilde{\omega} \subset \subset \omega^{*} \subset \subset \omega$ and let $\chi^{*}=\chi^{*}(x)$ be a new cut-off function, with $\chi^{*} \in C_{0}^{\infty}\left(\omega^{*}\right), \chi^{*} \geq 0$ and $\chi^{*} \equiv 1$ in $\tilde{\omega}$. Then, for some integer $m^{*} \geq m(k, j)$, one has

$$
\begin{aligned}
& \iint_{\omega(k, j) \times(0, T)}(t(T-t))^{-m(k, j)} \rho^{-2 s}|\Delta \phi|^{2} \\
& \leq \iint_{\omega^{*} \times(0, T)}(t(T-t))^{-m(k, j)} \rho^{-2 s} \chi^{*}|\Delta \phi|^{2} \\
& =\iint_{\omega^{*} \times(0, T)}(t(T-t))^{-m(k, j)} \rho^{-2 s} \chi^{*} \phi(-\Delta)^{2} \phi+\ldots \\
& \leq \iint_{\omega^{*} \times(0, T)}(t(T-t))^{-m^{*}} \rho^{-2 s} \chi^{*}|\phi|^{2}+C I_{12}(s, \lambda ; \Delta \phi)+\ldots \\
& \leq \iint_{\omega^{*} \times(0, T)}(t(T-t))^{-m^{*}} \rho^{-2 s} \chi^{*}|\phi|^{2} \\
& \quad+C \iint_{\omega^{*} \times(0, T)}(t(T-t))^{-m(1,0)} \rho^{-2 s}|\phi|^{2}+\ldots,
\end{aligned}
$$

where the dots denote again lower order terms. It is thus clear that there exist $m(k+1, j), C(k+1, j)$ and $\omega(k+$ $1, j$ ) such that

$$
I_{12}\left(s, \lambda ;(-\Delta)^{k+1} \partial_{t}^{j} \phi\right) \leq C(k+1, j) \iint_{\omega(k+1, j) \times(0, T)}(t(T-t))^{-m(k+1, j)} \rho^{-2 s}|\phi|^{2} .
$$

This ends the proof of the lemma.

\section{FURTHER COMMENTS AND OPEN QUESTIONS}

This section is devoted to make some comments on extensions of the previous results and, also, to report some related open problems.

The first question is whether the hypothesis (1.3) can be eliminated or at least weakened in Theorems 1.1 and 1.4. This is not clear; of course, as noticed in Remark 2.4, without new tools for the proof of (2.13), different from global (scalar) Carleman estimates, it seems very difficult to obtain the same results for $d_{\max } \geq 5$. In the one-dimensional case, when the matrix $M$ does not depend on $t$, a possible alternative is the reformulation of the null controllability problem for (1.1) as a moment problem. This has been done in some recent papers (see for instance $[7,11,13,20]$ ) given boundary controllability characterizations for some coupled parabolic systems.

Recall that the analysis and methods in Section 2 can be performed for the more general coupled systems

$$
\begin{cases}y_{t}-A S y=M(x, t) y+\sum_{k=1}^{N} W^{k}(x, t) \partial_{k} y+v 1_{\omega} & \text { in } Q, \\ y=0 & \text { on } \Sigma, \\ y(x, 0)=y_{0}(x) & \text { in } \Omega\end{cases}
$$


and

$$
\begin{cases}y_{t}-A S y=M(x, t) y+\sum_{k=1}^{N} \partial_{k}\left(W^{k}(x, t) y\right)+v 1_{\omega} & \text { in } Q, \\ y=0 & \text { on } \Sigma, \\ y(x, 0)=y_{0}(x) & \text { in } \Omega,\end{cases}
$$

where $A$ satisfies (1.2) and

$$
d_{\max } \leq 2
$$

$S$ is a second-order partial differential operator of the form

$$
S z:=\sum_{i, j=1}^{N} a_{i j}(x, t) \partial_{i} \partial_{j} z
$$

with $a_{i j}=a_{j i} \in W^{1, \infty}(Q)$ for all $i, j$ and

$$
\sum_{i, j=1}^{N} a_{i j}(x, t) \eta_{i} \eta_{j} \geq a_{0}|\xi|^{2} \quad \forall \eta \in \mathbb{R}^{N}, \quad \forall(x, t) \in Q, \quad a_{0}>0
$$

and $W^{k} \in L^{\infty}\left(Q ; \mathcal{L}\left(\mathbb{R}^{n} ; \mathbb{R}^{n}\right)\right)$ for all $k=1, \ldots, N$.

This also leads to controllability results for semilinear systems of a more general class than (1.11). More precisely, let us consider the system

$$
\begin{cases}y_{t}-A \Delta y=F(y, \nabla y)+v 1_{\omega} & \text { in } Q, \\ y=0 & \text { on } \Sigma, \\ y(x, 0)=y_{0}(x) & \text { in } \Omega,\end{cases}
$$

where $F: \mathbb{R}^{n} \times \mathbb{R}^{n \times N} \mapsto \mathbb{R}^{n}$ is given. We then have the following result:

Theorem 5.1. Let $A$ and $B$ be as in Theorem 1.1 and asume that (1.2) and (5.1) hold and

$$
F: \mathbb{R}^{n} \times \mathbb{R}^{n \times N} \mapsto \mathbb{R}^{n} \text { is globally Lipschitz-continuous. }
$$

Then (5.2) is exactly controllable to the trajectories.

The proof is similar to the proof of Theorem 1.4. The details are left to the reader; they rely on the ideas in [30], see the proof of Theorem 1.1 in [17].

Of course, it is again unknown whether the assumption $d_{\max } \leq 2$ can be suppressed.

For general linear systems of the kind (1.1), it is an open question to characterize those $n^{\prime} \leq n$ and $B \in$ $\mathcal{L}\left(\mathbb{R}^{n^{\prime}} ; \mathbb{R}^{n}\right)$ such that null controllability holds. Up to now, this is known only for constant matices $M$, as indicated in Theorem 1.5; see Remarks 1.2 and 1.3; see also [1,2] for some results in this direction and the recent paper [16], where the authors have introduced other techniques that could shed some light to this question.

It is also meaningful to consider boundary controllability problems for systems similar to (1.1) and (1.11). For instance, it makes sense to analyze the null controllability of

$$
\begin{cases}y_{t}-A \Delta y=M(x, t) y & \text { in } Q \\ y=B v 1_{\gamma} & \text { on } \Sigma, \\ y(x, 0)=y_{0}(x) & \text { in } \Omega,\end{cases}
$$

where $\gamma \subset \partial \Omega$ is a non-empty set, with controls $v$ for instance in $L^{\infty}\left(\gamma \times(0, T) ; \mathbb{R}^{n}\right)$. It also makes sense to analyze the exact controllability to the trajectories for the semilinear system

$$
\begin{cases}y_{t}-A \Delta y=f(y) & \text { in } Q \\ y=B v 1_{\gamma} & \text { on } \Sigma \\ y(x, 0)=y_{0}(x) & \text { in } \Omega\end{cases}
$$


When $n^{\prime} \geq n$ and $\operatorname{rank} B=n$, it is not difficult to deduce the null controllability of (5.3) and the exact controllability to the trajectories of (5.4) respectively from Theorems 1.1 and 1.4 (under similar assumptions for $A, M$ and/or $f$ ). However, when $n^{\prime}<n$, this is a much more complex question. Almost nothing is known in this context and, in general, the null controllability of (5.3) is an open question; see however $[1,2,7,13,20]$, for some particular results. As we said before, when $n^{\prime}<n$, even when the coupling matrix $M$ has constant coefficients, a minimal time of controllability $T_{0}=T_{0}(A) \in[0, \infty]$ for system (5.3) can appear (see [9]).

\section{REFERENCES}

[1] F. Alabau-Boussouira, Insensitizing exact controls for the scalar wave equation and exact controllability of 2-coupled cascade systems of PDE's by a single control. Math. Control Signals Systems 26 (2014) 1-46.

[2] F. Alabau-Boussouira and M. Léautaud, Indirect controllability of locally coupled wave-type systems and applications. J. Math. Pures Appl. 99 (2013) 544-576.

[3] F. Ammar-Khodja, A. Benabdallah and C. Dupaix, Null controllability of some reaction-diffusion systems with one control force. J. Math. Anal. Appl. 320 (2006) 928-943.

[4] F. Ammar-Khodja, A. Benabdallah, C. Dupaix and M. González-Burgos, A generalization of the Kalman rank condition for time-dependent coupled linear parabolic systems. Differ. Equ. Appl. 1 (2009) 427-457.

[5] F. Ammar-Khodja, A. Benabdallah, C. Dupaix and M. González-Burgos, A Kalman rank condition for the localized distributed controllability of a class of linear parabolic systems. J. Evol. Equ. 9 (2009) 267-291.

[6] F. Ammar-Khodja, A. Benabdallah, C. Dupaix and I. Kostin, Controllability to the trajectories of phase-field models by one control force. SIAM J. Control Optim. 42 (2003) 1661-1680.

[7] F. Ammar-Khodja, A. Benabdallah, M. González-Burgos and L. de Teresa, The Kalman condition for the boundary controllability of coupled parabolic systems. Bounds on biorthogonal families to complex matrix exponentials. J. Math. Pures Appl. 96 (2011) 555-590.

[8] F. Ammar-Khodja, A. Benabdallah, M. González-Burgos and L. de Teresa, Recent results on the controllability of coupled parabolic problems: a survey. Math. Control Relat. Fields 1 (2011) 267-306.

[9] F. Ammar-Khodja, A. Benabdallah, M. González-Burgos and L. de Teresa, A new relation between the condensation index of complex sequences and the null controllability of parabolic systems. C. R. Math. Acad. Sci. Paris 351 (2013) 19-20, 743-746.

[10] F. Ammar-Khodja, A. Benabdallah, M. González-Burgos and L. de Teresa, Minimal time of controllability of two parabolic equations with disjoint control and coupling domains. C. R. Math. Acad. Sci. Paris 352 (2014) 391-396.

[11] F. Ammar-Khodja, A. Benabdallah, M. González-Burgos and L. de Teresa, Minimal time for the null controllability of parabolic systems: the effect of the condensation index of complex sequences. J. Funct. Anal. 267 (2014) 2077-2151.

[12] J.-P. Aubin, L'analyse non linéaire et ses motivations économiques. Collection Mathématiques Appliquées pour la Maîtrise. Masson, Paris (1984).

[13] A. Benabdallah, F. Boyer, M. González-Burgos and G. Olive, Sharp estimates of the one-dimensional boundary control cost for parabolic systems and application to the $N$-dimensional boundary null-controllability in cylindrical domains. SIAM J. Control Optim. 52 (2014) 2970-3001.

[14] O. Bodart, M. González-Burgos and R. Pérez-García, Insensitizing controls for a heat equation with a nonlinear term involving the state and the gradient. Nonlin. Anal. 57 (2004) 687-711.

[15] F. Boyer and G. Olive, Approximate controllability conditions for some linear 1D parabolic systems with space-dependent coefficients. Math. Control Relat. Fields 4 (2014) 263-287.

[16] J.-M. Coron and P. Lissy, Local null controllability of the three-dimensional Navier-Stokes system with a distributed control having two vanishing components. Invent. Math. 198 (2014), no. 3, 833-880.

[17] A. Doubova, E. Fernández-Cara, M. González-Burgos and E. Zuazua, On the controllability of parabolic systems with a nonlinear term involving the state and the gradient. SIAM J. Control Optim. 41 (2002) 798-819.

[18] C. Fabre, J.-P. Puel and E. Zuazua, Approximate controllability of the semilinear heat equation. Proc. Roy. Soc. Edinburgh Sect. A 125 (1995) 31-61.

[19] H.O. Fattorini and D.L. Russell, Exact controllability theorems for linear parabolic equations in one space dimension. Arch. Rational Mech. Anal. 43 (1971) 272-292.

[20] E. Fernández-Cara, M. González-Burgos and L. de Teresa, Boundary controllability of parabolic coupled equations. J. Funct. Anal. 259 (2010) 1720-1758.

[21] E. Fernández-Cara, M. González-Burgos, S. Guerrero and J.-P. Puel, Exact controllability to the trajectories of the heat equation with Fourier boundary conditions: the semilinear case. ESAIM: COCV 12 (2006) 466-483.

[22] E. Fernández-Cara and E. Zuazua, The cost of approximate controllability for heat equations: the linear case. Adv. Differ. Equ. 5 (2000) 465-514.

[23] E. Fernández-Cara and E. Zuazua, Null and approximate controllability for weakly blowing up semilinear heat equations. Ann. Inst. Henri Poincaré Anal. Non Linéaire 17 (2000) 583-616.

[24] E. Fernández-Cara and S. Guerrero, Global Carleman inequalities for parabolic systems and applications to controllability. SIAM J. Control Optim. 45 (2006) 1399-1446.

[25] X. Fu, Null controllability for the parabolic equation with a complex principal part. J. Funct. Anal. 257 (2009) $1333-1354$. 
[26] A. Fursikov and O. Yu. Imanuvilov, Controllability of Evolution Equations, Vol. 34 of Lect. Notes Ser. Seoul National University, Research Institute of Mathematics, Global Analysis Research Center, Seoul (1996).

[27] M. González-Burgos and R. Pérez-García, Controllability results for some nonlinear coupled parabolic systems by one control force. Asymptot. Anal. 46 (2006) 123-162.

[28] M. González-Burgos and L. de Teresa, Controllability results for cascade systems of $m$ coupled parabolic PDEs by one control force. Port. Math. 67 (2010) 91-113.

[29] S. Guerrero, Null controllability of some systems of two parabolic equations with one control force. SIAM J. Control Optim. 46 (2007) 379-394.

[30] O. Yu. Imanuvilov and M. Yamamoto, Carleman inequalities for parabolic equations in Sobolev spaces of negative order and exact controllability for semilinear parabolic equations. Publ. Res. Inst. Math. Sci. 39 (2003) 227-274.

[31] G. Lebeau and L. Robbiano, Contrôle exact de l'équation de la chaleur. Comm. Partial Differ. Equ. 20 (1995) $335-356$.

[32] D. L. Russell, Controllability and stabilizability theory for linear partial differential equations: recent progress and open questions. SIAM Rev. 20 (1978) 639-739.

[33] I. Steinbach and F. Pezzolla, A generalized field method for multiphase transformations using interface fields, Physica. D 134 (1999) 385-393.

[34] I. Steinbach, F. Pezzolla, B. Nestler, M. SeeBelger, R. Prieler, G.J. Schimitz and J.L.L. Rezende, A phase field concept for multiphase systems. Physica D 94 (1996) 135-147.

[35] L. de Teresa, Insensitizing controls for a semilinear heat equation. Comm. Partial Differ. Eq. 25 (2000) 39-72. 